AAS 13-306

\title{
ASSESSMENT OF THE RECONSTRUCTED AERODYNAMICS OF THE MARS SCIENCE LABORATORY ENTRY VEHICLE
}

\author{
Mark Schoenenberger* \\ John Van Norman ${ }^{\dagger}$ \\ Artem Dyakonov \\ Chris Karlgaard $\$$ \\ David Way \\ Prasad Kutty\|
}

\begin{abstract}
On August 5, 2012, the Mars Science Laboratory entry vehicle successfully entered Mars atmosphere, flying a guided entry until parachute deploy. The Curiosity rover landed safely in Gale crater upon completion of the Entry Descent and Landing sequence. This paper compares the aerodynamics of the entry capsule extracted from onboard flight data, including Inertial Measurement Unit (IMU) accelerometer and rate gyro information, and heatshield surface pressure measurements. From the onboard data, static force and moment data has been extracted. This data is compared to preflight predictions. The information collected by MSL represents the most complete set of information collected during Mars entry to date. It allows the separation of aerodynamic performance from atmospheric conditions. The comparisons show the MSL aerodynamic characteristics have been identified and resolved to an accuracy better than the aerodynamic database uncertainties used in preflight simulations. A number of small anomalies have been identified and are discussed. This data will help revise aerodynamic databases for future missions and will guide computational fluid dynamics (CFD) development to improved prediction codes.
\end{abstract}

\section{INTRODUCTION}

On August 5, 2012, The Mars Science Laboratory entry vehicle (EV) successfully entered Mars atmosphere and landed the Curiosity rover safely on its surface. Data collected from on-board instrumentation was used to reconstruct the entry trajectory and measure information about the atmosphere encountered during its deceleration to parachute deployment. The Mars Entry Atmospheric Data System (MEADS) experiment flew aboard the MSL EV. Part of the Mars Entry Descent and Landing Instrumentation project (MEDLI), the MEADS experiments measured pressures at seven locations on the MSL heatshield. The pressure data was used to extract dynamic pressure, angle of attack and angle of sideslip and help determine Mach number. MSL carried an inertial measurement unit (IMU) that was used for navigation as the EV flew a guided entry to landing. Post-flight, the IMU measurements of accelerations and rates that were used to reconstruct the capsule's inertial velocity, position, and attitude history. The MSL aerodynamics database was also used to compare with IMU accelerometer measurements to provide a somewhat independent assessment of the wind-relative attitude during entry. The different data products have been used to reconstruct the MSL trajectory individually and then combined into a reconstruction with an Extended Kalman Filtering approach. The results of the reconstruction effort, for the first time at Mars, provide sufficient data to extract aerodynamic force and moment data, separated from the dynamic pressure experienced during entry. Recent capsules flown at Mars, with no surface pressure measurements, could only identify the net forces and moments acting on the vehicle but could not separate

\footnotetext{
* MSL aerodynamics Lead, NASA Langley Research Center, Hampton VA, 23681

${ }^{\dagger}$ MSL aerodynamics team, Analytical Mechanics Associates (at NASA Langley)

${ }^{\ddagger}$ MSL aerodynamics team, NASA Langley Research Center

$\S$ MEADS reconstruction lead, Analytical Mechanics Associates

"MSL flight mechanics lead, NASA Langley Research Center

" MEADS reconstruction analyst, Analytical Mechanics Associates
} 
aerodynamic performance from atmospheric density. The Viking landers [1,2] did have forebody pressure measurements during entry, but the attitude reconstructions from those missions were not satisfactory for a proper assessment of the aerodynamic database.

This paper will provide an overview of the preflight MSL aerodynamic database and compare that database with the reconstructed aerodynamic performance observed during flight. The dynamic pressure history measured during entry is used to extract the static force and moment coefficients. The preflight aerodynamic predictions, used for all mission design and flight operations simulations, are compared to the reconstructed aerodynamic coefficients. Assessments of notable differences and general agreement between preflight and reconstructed static forces and moments will be presented along with more qualitative assessments of the reaction control system (RCS) performance and dynamic stability. The aerodatabase uncertainty model is described and best-fit uncertainties on the reconstructed coefficients are estimated to help assess the accuracy of the preflight predictions. The local pressure measurements observed by the MEADS experiment will be compared with the closest computational predictions and the net forces and moments predicted by those solutions. The differences between the MEADS-converged attitude and forces and moments, and the corresponding aerodynamic forces and moments predicted by the MSL aerodynamc database will be compared and discussed.

\section{MSL Entry Vehicle}

To decelerate from entry velocity down to parachute deploy conditions the MSL mission used a blunt body entry capsule. The Mars Science Laboratory entry vehicle was comprised of a $70^{\circ}$ sphere-cone heatshield and backshell consisting of three truncated cone sections. The forebody was very similar to the heatshield geometry developed for the Viking Mars landers[3]. The backshell configuration is also very similar to Viking with a third cone section added to accommodate the volume of the parachute. The measured capsule diameter was 4.519 meters; it is the largest entry vehicle ever flown at Mars. During most of entry, the capsule used a radial cg offset to fly at an angle of attack (approximately $-16^{\circ}$ at hypersonic conditions). This attitude produced lift that was used to fly a guided entry profile, reducing the landing footprint to a much smaller sized than any previous Mars mission. To fly the guided entry, the MSL EV carried four pairs of 68lbf (nominal) reaction control system (RCS) jets to perform maneuvers and damp rates. The four pairs of jets could be fired rapidly in different combinations to provide control torques about roll, pitch, yaw, or any other axis by modulating the pulses of the jet.

Figure 1 shows a simplified version of the MSL capsule, representative of the flight vehicle and those used to populate the aerodynamic database with computational fluid dynamics (CFD) codes. The aerodynamic database CFD calculations were run on grids with geometry definitions based on a CAD models developed for earlier version of the heatshield. The flight capsule shoulder radius varied earlier configurations and the maximum diameter increased by about $19 \mathrm{~mm}$ due to an increase in heatshield thickness during the vehicle development process. A number of studies were conducted to assess the sensitivity to these shape changes and all were found to produce negligible changes on the aerodynamic predictions. The simplified geometry in Figure lis an accurate description of the flight geometry for aerodynamic modeling. Two sets of reference dimensions are given. The nominal values are those carried during MSL development and represent the original heatshield dimensions used for aerodynamic database grid generation. The second set are the measured dimensions. The aerodynamic coefficients presented and discussed in this paper are consistent with the measured values listed in Figure 1.

Figure 2 shows the aerodynamic force and moment definitions, and the design and measured centers of gravity location in the aerodynamic coordinate frame. The coordinate system origin is defined as the capsule nose. The cg location is defined in the aerodynamic coordinate frame relative to the nose. The center of gravity was offset from the capsule axis of symmetry to fly at an angle of attack to produce lift used to fly a guided entry. The aerodynamic coordinates are the standard convention for defining aircraft coefficients aligned to the capsule body $\left(C_{A}, C_{N}\right)$ rather than velocity vector $\left(C_{L}, C_{D}\right)$. The aerodynamic coefficients shown in Figure 2 are defined as

$$
\begin{aligned}
\text { Forces : } \quad C_{X} & =\frac{F_{X}}{q_{\infty} S_{r e f}} \\
\text { Moments : } \quad C_{x} & =\frac{M_{x}}{q_{\infty} S_{r e f} d_{r e f}}
\end{aligned}
$$




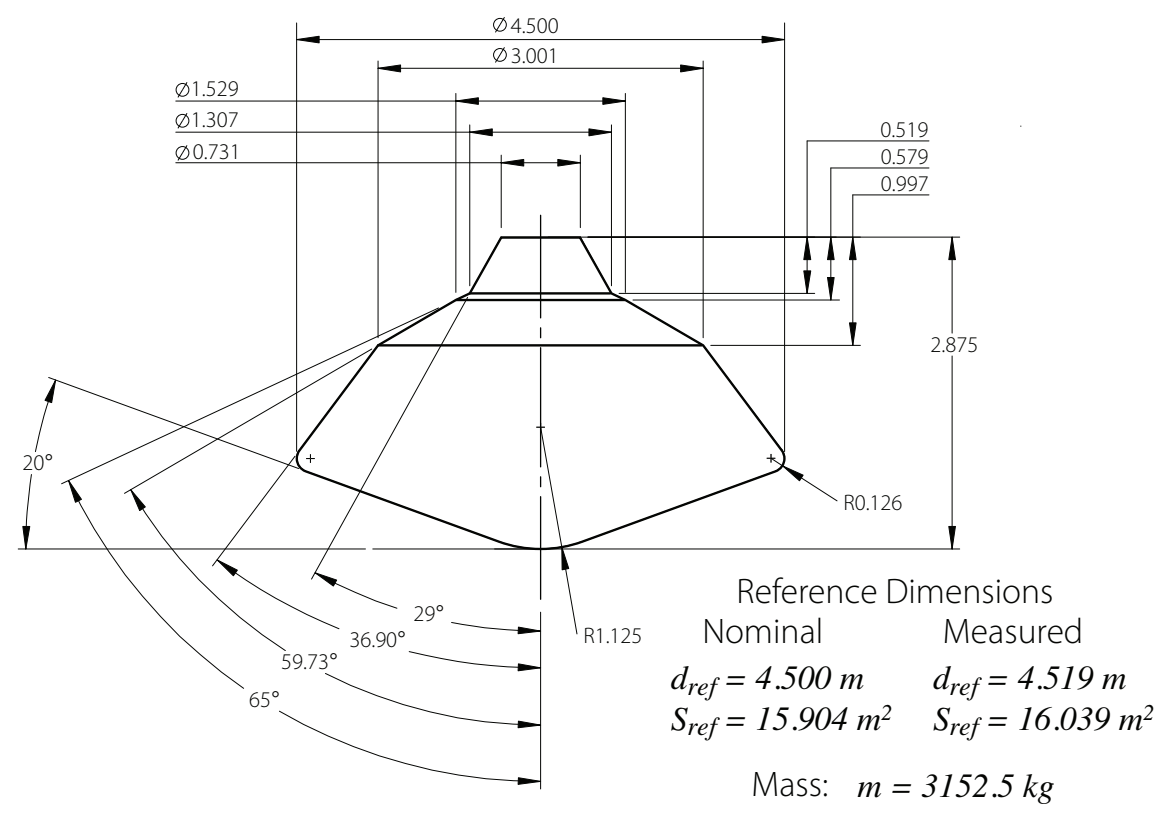

Figure 1. Mars Science Laboratory entry vehicle nominal dimensions (meters)

where $F_{X}$ and $M_{x}$ are the aerodynamic forces and moments acting on the capsule, $q_{\infty}$ is the freestream dynamic pressure and $S_{r e f}$ and $d_{r e f}$ are the measured reference area and diameter of the MSL EV.

\section{Entry Trajectory}

This work focused on assessing the aerodynamic performance of the MSL entry vehicle and comparing that performance to preflight predictions. The vehicle passed through many phases of flight after the entry vehicle served its function of slowing the payload from approximately $6000 \mathrm{~m} / \mathrm{s}$ down to a safe parachute deploy condition (near Mach 1.7). Just prior to parachute deploy (near Mach 2.0) a series of six balance masses were jettisoned to eliminate the radial cg offset, trimming the capsule near $0^{\circ}$ angle-of-attack for safe parachute deployment. During this mass jettison, the vehicle was also banked $180^{\circ}$ from its entry attitude to place the landing radar at the proper attitude following parachute deployment. The jettison of balance masses and the bank maneuver are collectively (and colloquially) referred to as the "straighten up and flight right" (SUFR) maneuver. The initiation of this maneuver (marked by the first balance mass jettison) will be noted in plots within this paper as the RCS firing and mass jettisons introduce vibrations and accelerations that influenced IMU and pressure measurements.

After parachute deploy, the vehicle decelerated to a safe velocity for the MSL rover, with its propulsive descent stage, to drop from the backshell and enter a powered flight mode. Using eight decent engines to provide deceleration thrust and full 6-degree-of-freedom control, the powered descent vehicle then diverted from the flight path of the parachute-backshell system an proceeded to descend to just above the Mars surface where the rover was lowered on tethers to a safe landing in a maneuver called "skycrane." In general, MSL flew very well through all phases of entry, descent and landing. The assessment the flight after capsule flight are documented elsewhere [4] and will not be discussed further here.

Figure 3 shows two representative MSL entry trajectories compared to the trajectory from which the MSL conditions were taken for aerodatabase CFD computations (MSL 05-22). The Mars Exploration Rover design trajectory is shown for reference as well. These trajectories help visualize the path that the MSL capsule took during its descent and deceleration through the Martian atmosphere. The MSL capsule flew deeper into the atmosphere than did the nonlifting MER capsule. In addition to slowing the vehicle, MSL flew a guided entry, using lift to minimize downrange error and later correct heading errors to fly to a very small landing footprint. Flying deeper in the atmosphere provided greater dynamic pressure for more lift force to improve guided flight performance. The MSL vehicle lofted higher than the reference trajectory used for CFD conditions. Comparison of computations along both trajectories show that 


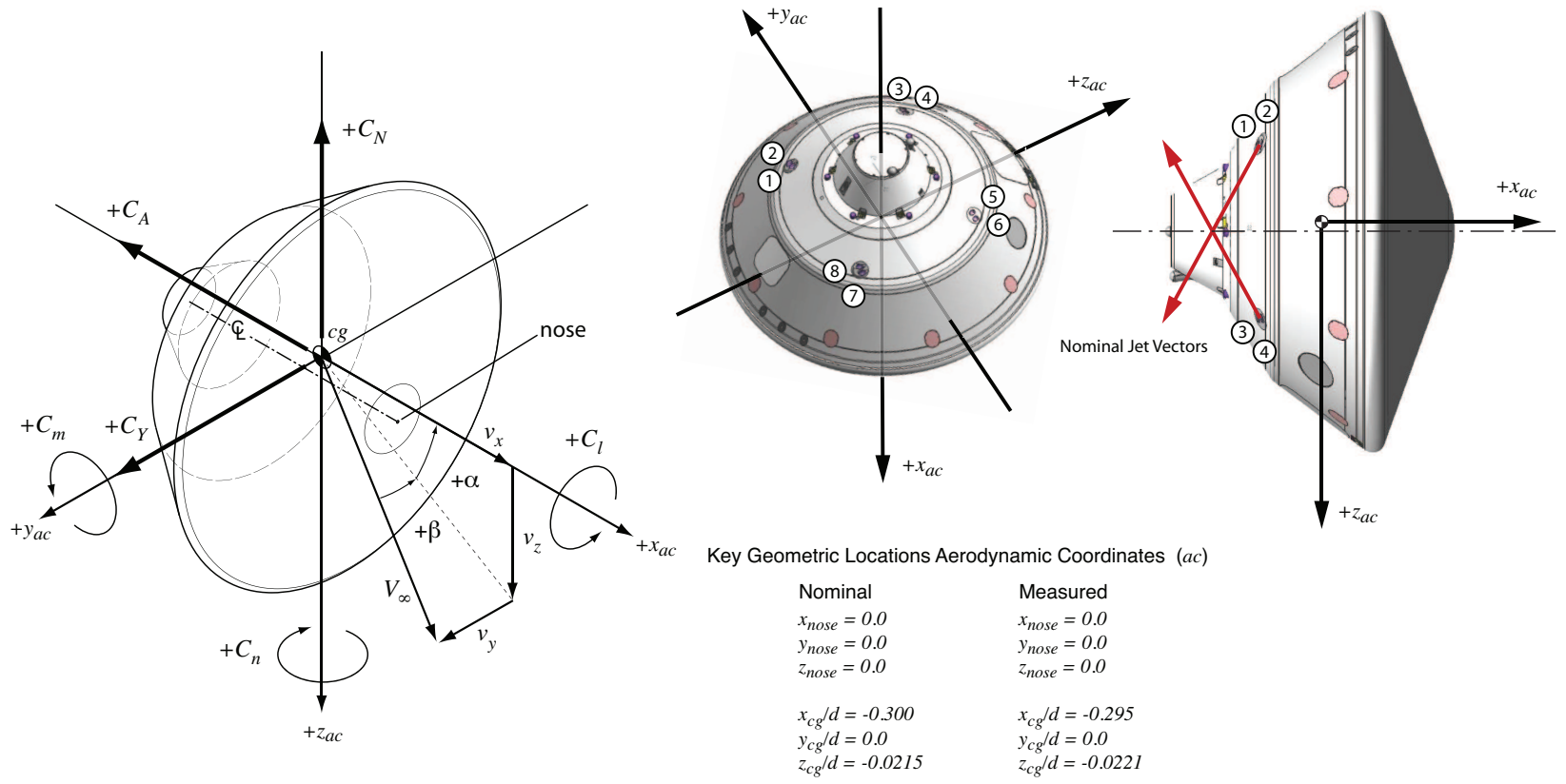

Figure 2. MSL aerodynamic and coordinate frame definitions

the errors due to CFD cases being run at conditions away from the actual flight profile are very small. Further analysis may be able to attribute the small differences between prediction and reconstructed aerodynamic coefficients to small differences identified in preflight uncertainty analysis. The potential sources of error include, trajectory conditions, turbulence models, real gas models in CFD and others. Engineering-judgment interpretations of observed differences will be provided here. Conclusively identifying the specific error sources that reconcile differences is beyond the scope of this work.

\section{MSL Aerodynamic Database}

The Langley Aerothermodynamic Upwind Relaxation Algorithm (LAURA) [5] CFD code was used to predict the static aerodynamic force and moment coefficients in the continuum flight regime. This data was used to design the MSL entry trajectory and during flight operations the data was used to target the final landing location. LAURA is a finite volume, shock capturing algorithm capable of calculating flows in chemical and thermal non-equilibrium. LAURA can be used for inviscid, thin-layer Navier-Stokes, or full Navier-Stokes computations. The solutions in the MSL aerodatabase were run with flags set for laminar and non-blowing conditions, and solved the thin-layer Navier-Stokes equations. The calculations were performed on Intel Linux clusters (32-bit word length) with LAURA version "LAURA_intel" (last modified March 17, 2006). The calculations for a given solution were spread over many processors via Message Passing Interface (MPI) [6].

The LAURA CFD matrix is shown in Figure 4. The conditions along the reference trajectory shown in Figure 3 were used as freestream conditions for the flight CFD calculations. More details of the MSL aerodynamic database are described in papers by Schoenenberger et al and Dyakonov et al [7,8]. The aerodynamic coefficients presented in this paper for comparison to the reconstructed aerodynamic coefficients derived from IMU and MEADS data were obtained using the same MSL aerodynamic database code as was used in preflight simulations (using reconstructed trajectory states). Note that CFD is used for all data points, but for all supersonic points noted in Figure 4, the backshell contribution is not accounted for using the CFD pressure distributions. Instead, the backshell contribution to axial force is modeled with a curve-fit derived from Viking flight data (backshell pressure measurements). The backshell contributions to normal and side force coefficients as well as pitch and yaw moments were found to be negligible and therefore $C_{A}$ is the only coefficient with a correction. The base correction model is presented in the paper by Dyakonov et al[8]. This methodology was developed for Mars Pathfinder and used again for the Mars Exploration 


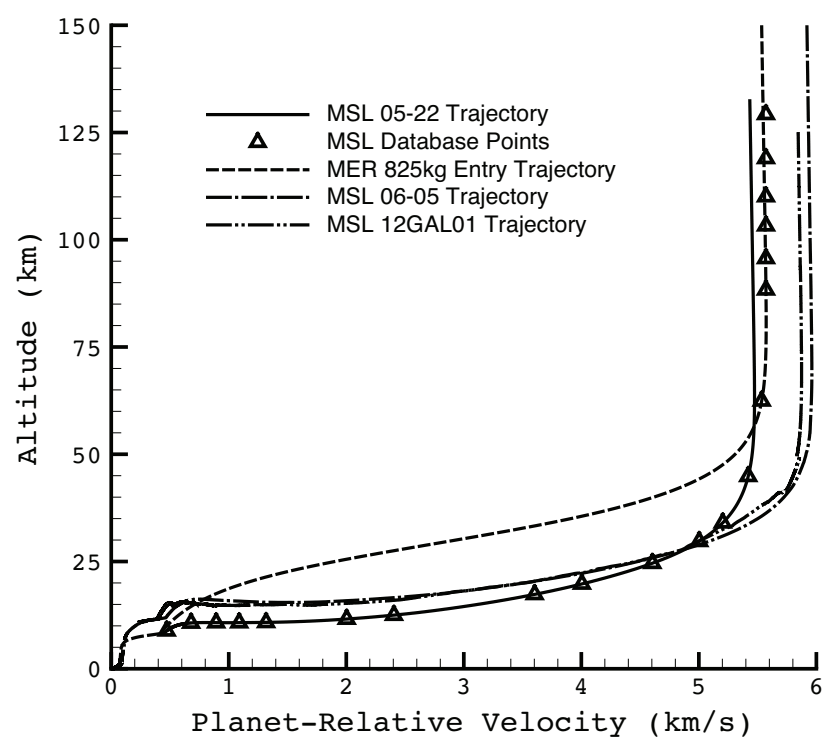

Figure 3. MSL Database points along design and reference trajectories

Rover and Mars Phoenix aerodynamic databases. The MEADS data is the first data set to provide any independent information to assess this base correction.

\section{METHODS}

To assess the preflight aerodynamic database and extract as much detail as possible in that assessment, an accurate reconstruction of the MSL entry trajectory is required. The path the EV followed during entry, its orientation and in a perfect world, full knowledge of atmosphere properties including wind velocity are required to extract the aerodynamic coefficients. The reconstruction efforts sponsored by the MEDLI project have developed the methodologies to use onboard instrumentation including MEADS pressure measurements and the descent inertial measurement unit (DIMU) to reconstruct the MSL capsule entry as accurately as possible and to extract aerodynamic coefficients for comparison to preflight predictions. Karlgaard et al $[9,10,11]$ have developed the MSL reconstruction methodology based on techniques developed for a number of recent flight projects, including Ares 1-X and Hyper-X. The reconstruction process and how the capsule state, aerodynamic performance and Mars atmosphere were reconstructed from inertial and pressure telemetry is described briefly below with details provided in cited papers. This section is intended to provide sufficient detail regarding the reconstruction process to understand the data being compared to preflight predictions.

Instrumentation Accurate reconstruction was possible for the MSL entry because of two key instruments. First the DIMU provided a set of angular rates and axial accelerations to reconstruct the inertial path and attitude during entry. Second, the MEADS experiment provided seven pressure measurements on the heatshield which served as a flush air data system (FADS) to measure the dynamic pressure that the capsule experienced during entry as well as the wind-relative angles of attack and sideslip, $\alpha$ and $\beta$.

Figure 5 shows the pressure port locations on the MSL heatshield and a drawing of an installed transducer. The port locations were selected to measure stagnation pressure and resolve angles of attack and sideslip. Ports 1 and 2 were located at or near the expected stagnation point during entry to provide an accurate stagnation pressure to reconstruct freestream dynamic pressure. Ports 6 and 7 are located off the nominal pitch plane to provide sideslip information. Ports 1 through 5 provide multiple data points to compare with CFD predictions to resolve angle of attack. The full scale range of the transducers was $5.0 \mathrm{psi}(34.474 \mathrm{kPa})$. The range was selected to ensure that no transducer overranged at peak dynamic pressure during entry, but still provided accurate dynamic pressure and attitude measurements down to $850 \mathrm{~Pa}$ (a MEADS design requirement). Pressure data was collected at $8.0 \mathrm{~Hz}$ through all of entry until just before heatshield jettison. Details of the hardware, its installation and calibration are summarized by Munk et al [12].

The MSL heatshield was built with an aluminum honeycomb structure covered with a thermal protection system 


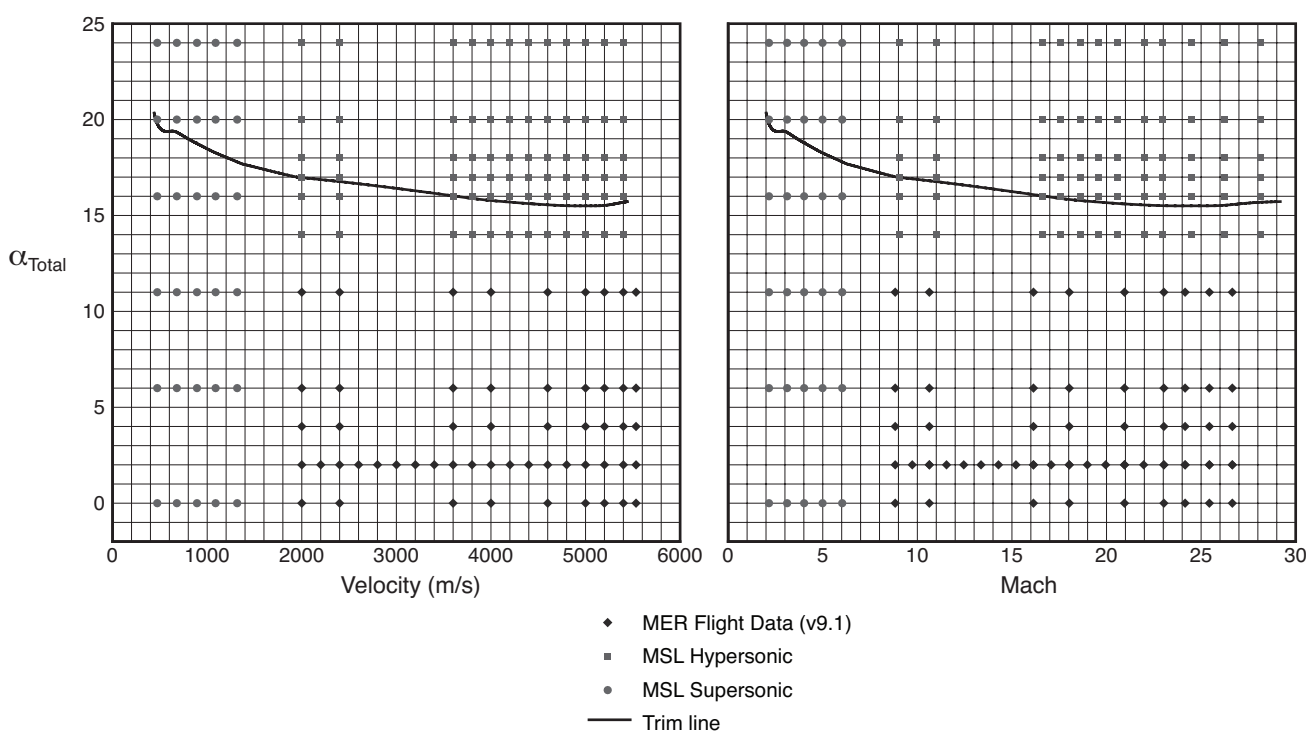

Figure 4. CFD Matrix in MSL preflight database (predicted trim angle shown)

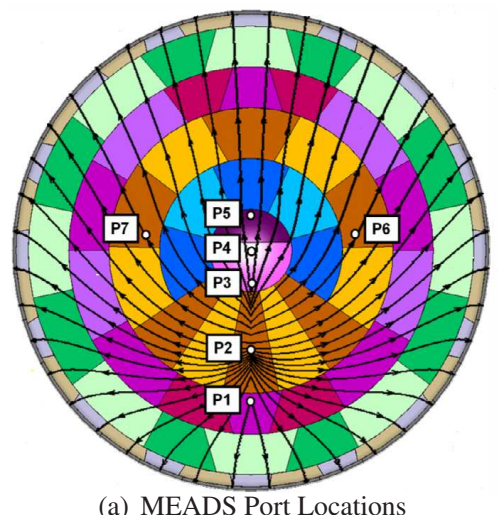

(a) MEADS Port Locations

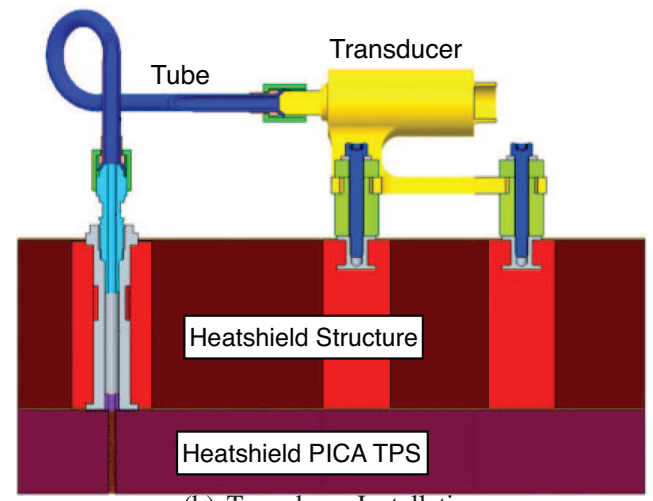

(b) Transducer Installation

Figure 5. MEADS layout and mechanical installation

(TPS) consisting of Phenolic Impregnated Carbon Ablator (PICA) tiles. MSL was the first vehicle flown at Mars with a tiled heatshield. Figure 5 shows the tile arrangement and the cross-section of the structure and TPS material. The gaps between tiles were filled with room temperature vulcanizing (RTV) sealant. Port locations were selected in-part to maintain "safe" distances from the gaps to avoid aerodynamic influences on pressure measurements from surface features associated with local ablation or roughness near the seams.

The DIMU used to provide navigation information during entry, descent and landing was a Honeywell Miniature Inertial Measurement Unit (MIMU)* The device measured angle and velocity increments across time steps, recording them at $200 \mathrm{~Hz}$. These increments were converted to angular rates (then quaternions) and accelerations to perform an inertial reconstruction of the capsule trajectory. The DIMU was installed to an angular accuracy of 1 milliradian about each axis relative to the descent stage. Through a stack-up of quality assurance (QA) measurements it was determined that the orientation knowledge of the DIMU relative to the heatshield surface was also on the order of 1 milliradian.

\footnotetext{
${ }^{*}$ Technical specifications at http://www51.honeywell.com/aero/common/documents/myaerospacecatalog-documents/MIMU.pdf
} 


\section{Reconstruction of MSL Entry Trajectory}

Papers by Karlgaard et al $[13,14]$ describe the preliminary reconstruction process and a preliminary Kalman Filter reconstruction using all available data sets. For this analysis, the DIMU and MEADS data were used together to estimate the best entry trajectory of the MSL entry capsule. The aerodynamic model reconciliation described in this paper uses two separate reconstruction methods to get an appreciation of the sensitivity of the extracted aerodynamic coefficients to the remaining unknowns in the reconstruction process (primarily winds and density). These techniques are semi-independent methods that utilize different portions of the entire data set. The first technique is a pure inertial solution, and the second approach makes primary use of the MEADS pressure measurements. This paper represents a preliminary assessment of the aerodynamic database. Future work will better resolve the reconstruction sources of error including wind and density errors which are not assessed in detail here.

The pure inertial reconstruction (referred to in this paper as the DIMU reconstruction) is based on a direct numerical integration of the measured accelerations and angular rates, using initial conditions obtained from orbit determination. An assumed atmosphere profile based on MSL-project mesoscale models was utilized to produce atmospheric-relative quantities such as Mach number and dynamic pressure. The angles of attack and sideslip are calculated from the aerodynamic axis velocities that result from the numerical integration of the DIMU accelerations and rates. It is important to note that zero winds relative to the planet was assumed.

The MEADS pressure reconstruction (referred to as the MEADS/DIMU reconstruction) utilizes a nonlinear leastsquares algorithm to produce estimates of angle of attack, angle of sideslip, dynamic pressure, and static pressure. The least-squares algorithm includes a novel IMU-aiding approach in which the IMU velocity magnitude is used to improve the estimate of Mach number. Atmospheric density is computed from the dynamic pressure estimate and the IMU velocity, assuming no winds. The MEADS dynamic pressure and IMU acceleration and angular rate measurements can also be combined to produce estimates of the vehicle aerodynamic forces and moments. It will be those reconstructed coefficients that are compared to preflight predictions below.

Figure 6 shows a comparison of the DIMU-reconstructed angles of attack and sideslip with the last preflight simulation before entry. The POST II [15] simulation, OD230, took the last orbit determination information prior to entry and latest mesocale atmosphere prediction to provide a final estimate of the entry performance before landing. These preflight predictions agree remarkably well with the reconstructed angles of attack and sideslip derived from the IMU. There are some deviations which will be discussed later, but this comparison indicated that in general the capsule behaved very much as predicted. Figure 7 shows the reconstruction of dynamic pressure and Mach number along the capsule entry trajectory using MEADS pressure measurements (anchored with the DIMU inertial velocity) and the DIMU measurements with a preflight atmosphere model. Both sets of data show very close agreement and the differences are within the uncertainties of the measurement instruments and preflight atmosphere model. Again, these comparisons show that the flight through the atmosphere was much as expected. These plots show the reconstructed state data that was used for aerodynamic parameter identification, enabling the assessment of the aerodynamic database. The capsule attitude, velocity and Mach number were used to query the aerodynamic database. The dynamic pressure with the DIMU acceleration measurements were used to extract the static aerodynamic coefficients from the flight telemetry (described below) to compare with the nominal database values.

Reaction Control Firing History Figure 8 shows plots of preflight simulation predictions of RCS firings and the commands given during flight. The roll pitch and yaw commands were calculated by summing all eight jets, assigning either a +1 or -1 contribution to that axis depending on the jet. For example a value of +4 for the roll axis would indicate that four of eight jets are commanded to provide a positive roll torque and the remaining four jets are inactive. Summing the the jets into these primary torque commands helps simplify the eight-jet firing history to see what actions the controller was requesting. The most notable events are the three bank reversals, indicated by a full bank command to start the rotation, immediately followed by a roll command of opposite sign to arrest the motion. Both the preflight and flight data indicate three reversals. In the flight data the first starts at $612 \mathrm{~s}$, followed shortly after by another around 633s. The third and final bank reversal occurs at 663s. The $180^{\circ}$ roll that occurs during SUFR is also evident starting at 780s. In general bank reversals in the flight data appear very similar to preflight predictions. The flight data shows less RCS activity away from the big bank commands than was predicted prior to entry. This will be discussed below.

Comparison of MEADS pressures to LAURA CFD solutions To better appreciate the accuracy of both the trajectory data and aerodynamic coefficients extracted using the MEADS pressure measurements it is important to compare the measured pressures to the "truth" model used to convert raw pressure measurements into the desired data products. 


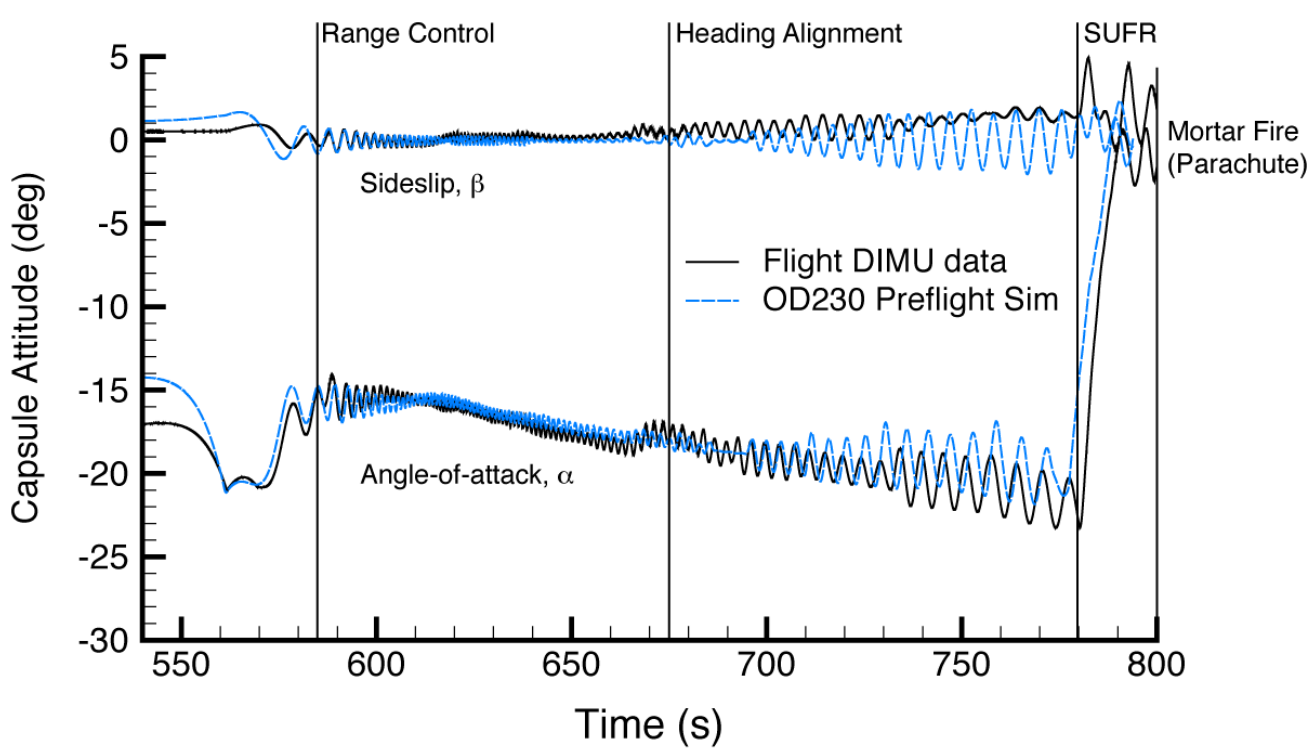

Figure 6. Comparison of preflight and reconstructed angles of attack and sideslip
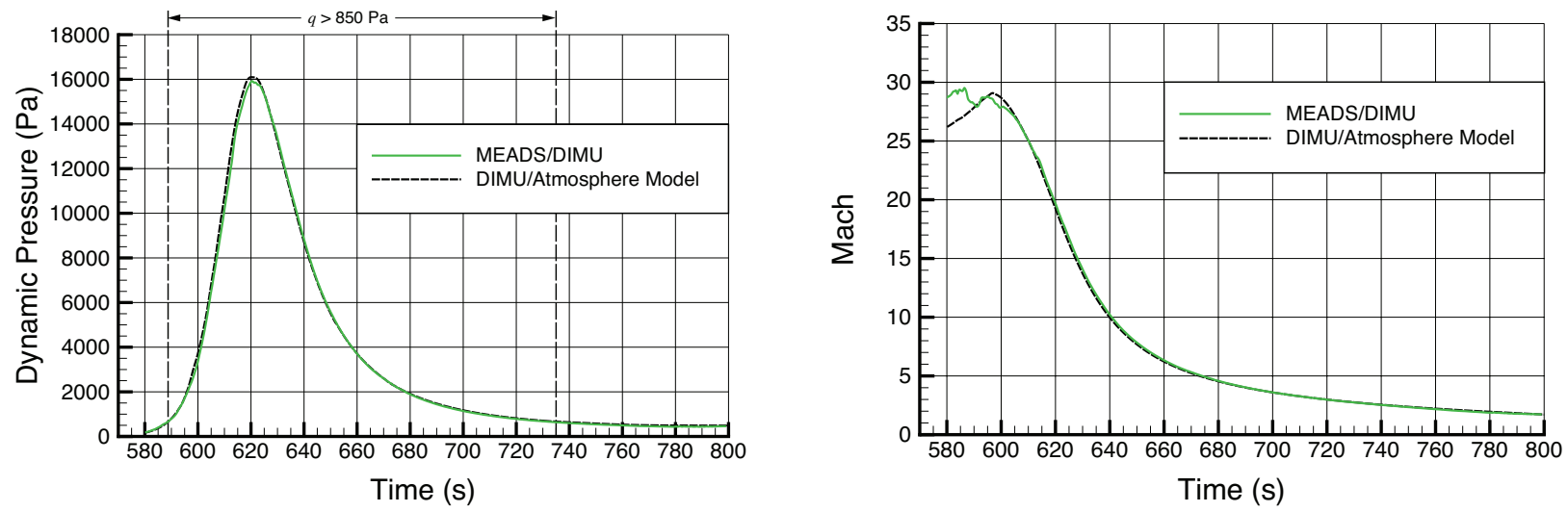

Figure 7. Reconstructed dynamic pressure and Mach number

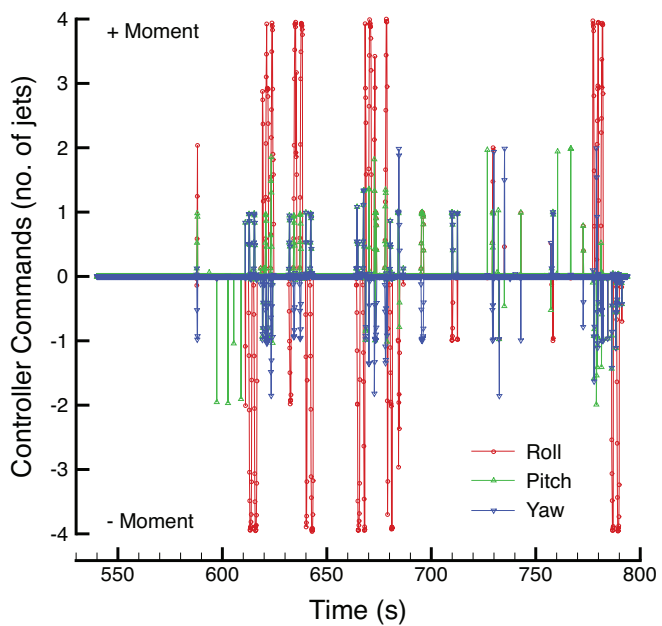

(a) OD230 Simulation Data

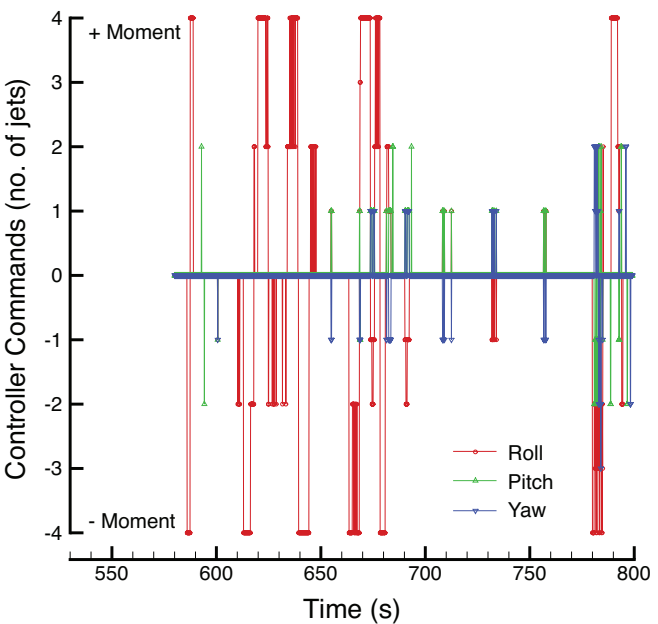

(b) Flight data

Figure 8. Comparison of RCS firing activity, last nominal simulation and flight data 
The "truth" model was built from surface pressure distributions from the LAURA CFD solutions used to generate the MSL aerodynamic database. The CFD solutions are the best predictions of the pressure distributions expected during entry through the Mars atmosphere. There is the potential for the aerodatabase coefficients and MEADS extracted parameters to be partially correlated as they both use the same sets of data. However, there are signficant differences in how the information is used in database construction and parameter identification, so that the results are largely independent. The MEADS reconstruction of aerodynamic coefficients uses CFD to determine dynamic pressure only (together with independent accelerometer measurements in the DIMU), given the measured stagnation pressure. Analysis has shown the LAURA CFD predictions of the ratio of dynamic to stagnation pressure accross then entry trajectory to be very accurate. The aerodynamic database integrates the full CFD pressure distribution and any errors away from the MEADS ports become errors in the database coefficients.

A comparison of the seven MEADS measurements to the CFD pressures at the converged state provides a qualitative and quantitative assessment of MEADS state measurements. Close agreement at each port location suggests that the flight pressure distribution is in good agreement with prediction. A large variation in fit quality among the MEADS pressures would suggest that the CFD pressure model did not properly predict the surface pressure distribution during flight, or that one or more MEADS transducers experienced some kind of anomaly. Figure 9 shows a comparison of measured pressures with the converged points in the CFD model. The pressure data are presented as a pressure coefficient, $C_{p}$, defined as

$$
C_{p}=\frac{p-p_{\infty}}{q_{\infty}}
$$

where $p$ is a MEADS pressure measurement or local CFD pressure value, $p_{\infty}$ is the local freestream pressure and $q_{\infty}$ is the local dynamic pressure. The CFD model is defined in terms of pressure coefficient. Non-dimensionalizing the absolute pressures eliminates variations due to trajectory, keeping the database a function of velocity or Mach and angles of attack and sideslip. Very large deviations from the expected trajectory would introduce some errors, but analysis of the aerodynamic database shows that such errors are small for the MSL preflight and flight trajectories. The two plots in Figure 9 are at supersonic and hypersonic conditions at angles of attack close to database CFD solutions. These plots are representative of the data fit quality during entry and shown as representative examples. Ports 6 and 7 are not shown but have similar agreement between measurement and the CFD model. The MEADS pressures follow the CFD pressure distribution very closely at both conditions. Port 5 has the only notable variation. That port is located at a spot on the heatshield that is subject to a local pressure drop as flow around the capsule nose accelerates around the sphere-cap to the lee-ward side of the forebody cone. The local pressure minimum does not remain at the same spot along the trajectory (as shown in these two plots). CFD errors in predicting this local nonlinear phenomenon appear to be the largest disagreement between flight measurements and the model. Figure 10 shows the pressure residuals (CFD pressure at the converged point subtracted from the measured pressures) for each transducer. The largest residuals occur at peak dynamic pressure as that is the time when peak absolute pressures are measured. Even there the residuals are generally under $100 \mathrm{~Pa}$ which is less than $1 \%$ of reading. Port 5 does appear to have the largest absolute residual near peak dynamic pressure, but agreement over the trajectory is similar to the other ports. More importantly there does not appear to be any systematic biases of any port. The Port 5 residual appears to drift linearly from $\sim 680-780$ seconds. Ports 6 and 7 both show an approximately sinusoidal residual variation across the peak dynamic pressure pulse ( 590-640s). These kinds of variation hint at how CFD mis-predicts the flight pressure distribution. The symmetry of the residual signal for ports 6 and 7 is particularly compelling. All variations are small, but this information may be used in future efforts to improve CFD codes by reconciling prediction with flight data. Overall, the agreement of MEADS and model pressures are excellent and add confidence in the reconstructed angles and dynamic pressure.

\section{Extraction of Aerodynamic Forces and Moments}

The dynamic pressure from the MEADS/IMU reconstructed trajectory $\left(q_{\infty}=q_{\infty, M E A D S}\right.$ was used to extract aerodynamic forces. The aerodynamic forces can be determined using the IMU accelerometer measurements, rotated into the aerodynamic coordinate system $\left(a_{x}, a_{y}\right.$, and $\left.a_{z}\right)$, with the measured entry vehicle mass. $m_{E V}$, and reference area, $S_{r e f}$.

$$
C_{A}=-\frac{m_{E V} a_{x}}{q_{\infty, M E A D S} S_{r e f}}
$$




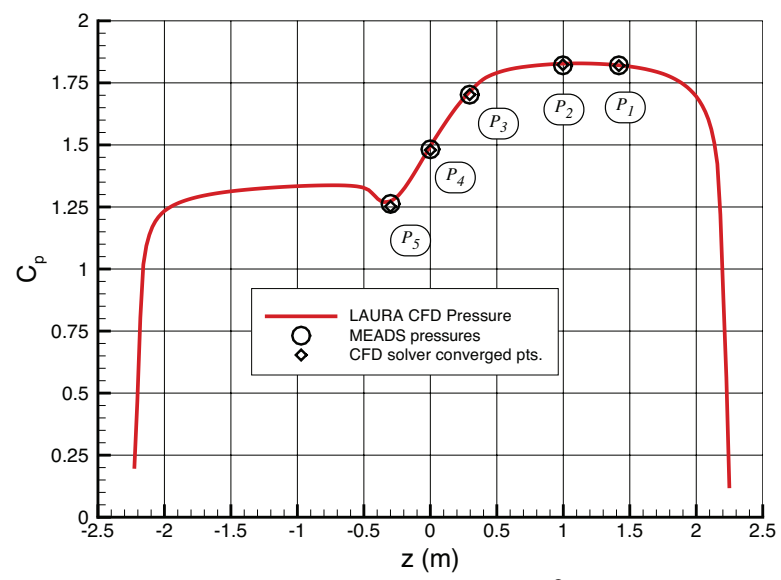

(a) $M_{\infty}=4.0, \alpha=-16.4^{\circ}$

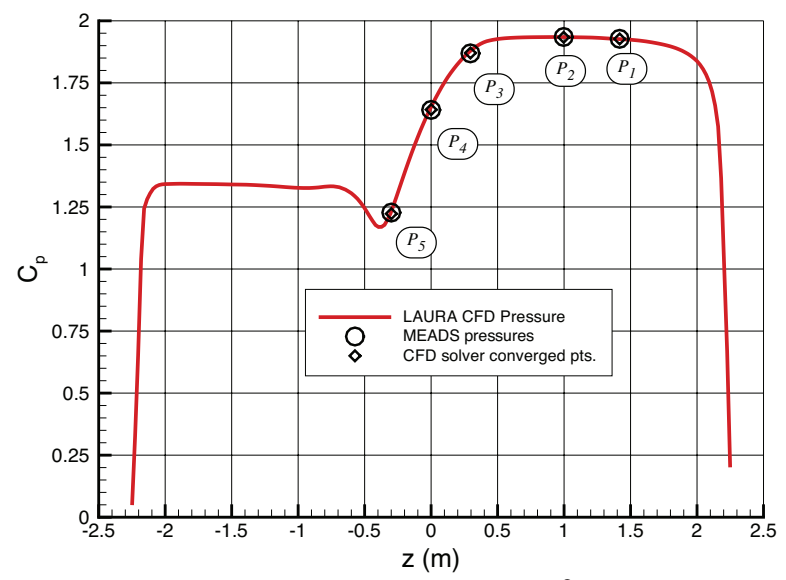

(b) $M_{\infty}=18.0, \alpha=-18.9^{\circ}$

Figure 9. MEADS pressures compared to CFD surface and converged CFD port values

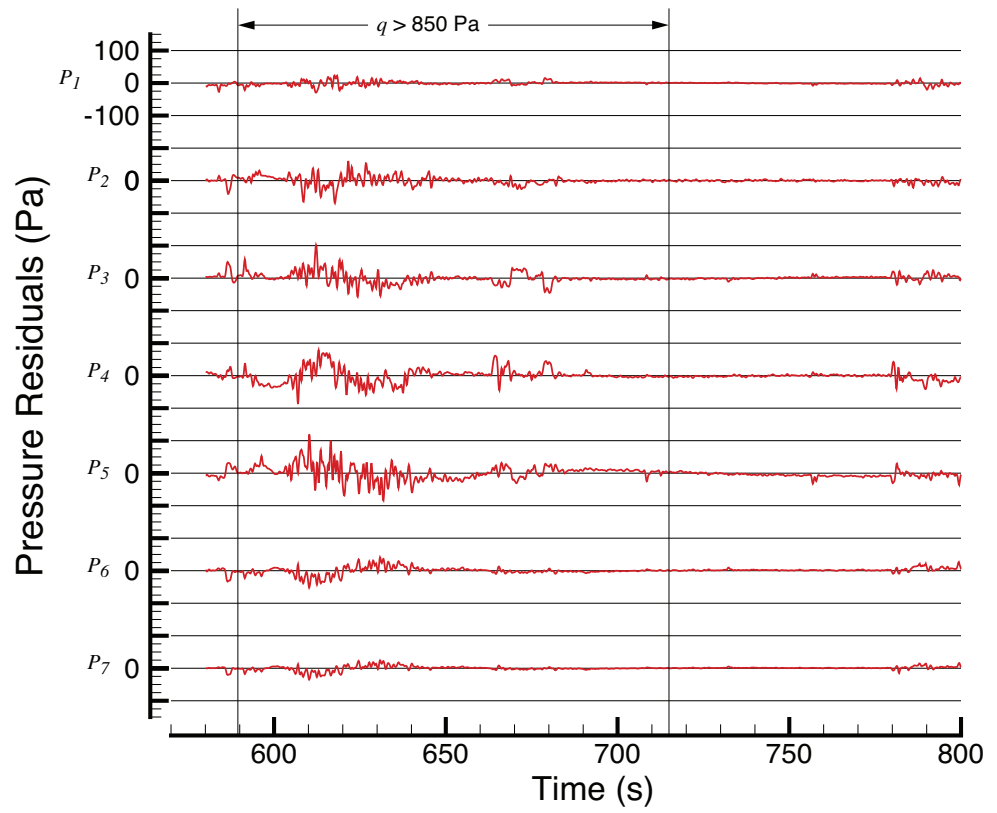

Figure 10. Residuals of MEADS pressures measurements with converged CFD pressure model 


$$
\begin{gathered}
C_{Y}=\frac{m_{E V} a_{y}}{q_{\infty, M E A D S} S_{r e f}} \\
C_{N}=-\frac{m_{E V} a_{z}}{q_{\infty, M E A D S} S_{r e f}}
\end{gathered}
$$

The mass listed in Figure 1 was the expected mass of the entry vehicle at entry interface based on pre-launch measurements. Prior to entry the RCS system is used to de-spin the vehicle and turn to the proper orientation for entry. After interface the RCS is used to perform bank reversals and damp rates. The commanded RCS firing history is known from telemetry but the fuel mass expended with each firing can only be estimated. The total RCS propellant mass used during capsule flight was estimated and used in the reconstruction process. The fuel usage during EV flight was very small ( $\sim 0.5 \%$ of the vehicle mass at entry) and a detailed mass accounting for each RCS firing was not done.

The static moments can be extracted from the flight telemetry and can also be assessed in by looking at the pitch and yaw frequencies of oscillation and the angle of attack history. First, the static moments can be measured using the IMU angular rate information.

$$
\mathbf{M}_{\text {aero }}=\mathbf{I} \overrightarrow{\dot{\omega}}+\vec{\omega} \times \mathbf{I} \vec{\omega}
$$

Where $\mathbf{I}$ is the measured moment of inertia tensor and $\vec{\omega}$ are the measured angular rates. The angular acceleration vector, $\overrightarrow{\dot{\omega}}$, were determined by taking finite difference derivatives of the DIMU angular rates. The moment coefficients can then be determined by non-dimensionalizing the measured moments by the MEADS/DIMU dynamic pressure and measured reference area and length.

$$
\begin{aligned}
C_{l} & =\frac{M_{\text {aero }, i}}{q_{\infty, M E A D S} S_{r e f} d_{r e f}} \\
C_{m} & =\frac{M_{a e r o, j}}{q_{\infty, M E A D S} S_{r e f} d_{r e f}} \\
C_{n} & =\frac{M_{a e r o, k}}{q_{\infty, M E A D S} S_{r e f} d_{r e f}}
\end{aligned}
$$

The capsule motion during entry showed very small oscillations in angle of attack and sideslip and virtually no roll that was not commanded by the onboard controller. The RCS frequently disturbed the natural capsule angular rates and accelerations as bank maneuvers and rate damping jet-firings were executed. Therefore, the moments measured directly from the rates and their derivatives are subject to significant errors. Also, as the moments are resolved about the center of gravity, the rate data does not provide information about the accuracy of preflight predictions of trim angle throughout entry.

Two other types of data comparisons were conducted to provide additional assessments of the MSL pitch and yaw stability predictions. First a simple comparison of the predicted trim angle to the reconstructed angle of attack history shows how well the preflight calculations predicted the static stability and how trim angle varies with radial cg offset. The predicted trim angle, using the measured cg location and nominal aerodynamic database will be compared to the reconstructed angle of attack history later in this document. Second, the frequencies of oscillation in pitch and yaw can be compared to the natural frequencies predicted by local static stability coefficients. Damping has a very small contribution to the natural frequency, so the predicted frequencies can be estimated by the following relations:

$$
\begin{gathered}
f_{y}=\frac{\omega_{y}}{2 \pi}=\frac{1}{2 \pi} \sqrt{-\frac{q_{\infty, M E A D S} S_{r e f} d_{r e f} C_{m_{\alpha}}}{I_{y y}}} \\
f_{z}=\frac{\omega_{z}}{2 \pi}=\frac{1}{2 \pi} \sqrt{-\frac{q_{\infty, M E A D S} S_{r e f} d_{r e f} C_{n_{\beta}}}{I_{z z}}}
\end{gathered}
$$


Where $f_{y}$ and $f_{z}$ are the pitch and yaw oscillation frequencies in Hertz. The derivatives, $C_{m_{\alpha}}$ and $C_{n_{\beta}}$ are the local pitch and yaw static stability slopes (per radian) determined from the MSL aerodynamic database. $I_{y y}$ and $I_{z z}$ are the measured moments of inertia about the pitch and yaw axes. The frequencies predicted by the nominal aerodynamic database were calculated using these equations, the measured mass properties and reference dimensions and the MEADS/DIMU dynamic pressure history. The frequencies were also extracted directly from the flight telemetry for a comparison. The capsule pitch and yaw frequencies (oscillation frequencies about the vehicle center of gravity) were extracted from a Time-Frequency (TF) analysis of the DIMU data. This analysis utilizes MATLAB routines from the Time-Frequency Toolbox (TFTB) ${ }^{\dagger \ddagger}$. This toolbox makes use of a TF method, referred to as "reassignment," which improves the readability of TF distributions [16]. The signals used in this analysis were yaw and pitch rates (about the body y- and z-axes) from the raw DIMU telemetry. The frequencies extracted from the flight telemetry are compared to the predicted frequencies described above. Signals were down-sampled by interpolation to regular $12 \mathrm{~Hz}$ intervals, allowing $4096\left(2^{12}\right)$ frequency bins over approximately $340 s$ of the trajectory, which is sufficient to capture the entire entry deceleration pulse (approximately $213 \mathrm{~s}$ ) as well as the full time on parachute (approximately $117 \mathrm{~s}$ ). The data presented here is for the capsule dynamics before parachute deploy. More details of this TF analysis are described in a paper by Way et al [17].

Uncertainty Analysis and Sources of Error A significant portion of the MEADS reconstruction development was the identification and quantification of the uncertainties in measuring the MEADS parameters. This activity included assessing pressure transducer and DIMU accuracy, installation tolerances, lag times and many others. A matrix of all error sources was established for the certification of the MEADS experiment for flight. It was shown that the installed instrumentation has the accuracy to resolve the dynamic pressure to within $2 \%$ angles of attack and sideslip to within $0.5^{\circ}$ (for $q_{\infty}>850 \mathrm{~Pa}$ ) assuming the CFD-based pressure model perfectly predicted the flight surface pressures. The largest source of uncertainty in the MEADS measurements was found to be the CFD pressure model used as truth to convert the measured pressures to angles and dynamic pressure. An extensive CFD calibration campaign, using wind tunne pressure tests, was undertaken to help quantify the local surface (MEADS port) uncertainties in CFD solutions. However, the test accuracy tended to be on the order of the uncertainties carried for the CFD solutions. While the testing could not reduce the pressure database uncertainty model, they did provide confirmation that the model was of the right order. CFD analysis was run to assess uncertainty effects due to grid resolution, heatshield ablation, deformation, OML shape knowledge and hole location knowledge error. It was found that for each port, the accuracy of the CFD database was good to about $0.5 \%$ of the measure pressure coefficient or less. The total error for each point is modeled in the MEADS reconstruction algorithm. In preflight analysis, the CFD pressure model dominated all hardware and installation sources of error. Figure 11 shows the $3 \sigma$ (three standard deviation) uncertainties from the MEADS/DIMU reconstruction for the portion of the trajectory where dynamic pressure is greater than $850 P a$. The curves shows the increase in uncertainty, above the instrumentation sytem uncertainties, due to the CFD uncertainty model. In practice, the good convergence of MEADS flight measurements to the CFD model suggest the preflight uncertainty models may be conservative. However, these uncertainty curves do provide a reasonable bound for the uncertainties of the reconstructed dynamic pressure, angle of attack and sideslip, and axial force coefficient. The dynamic pressure uncertainties remains fairly constant, between 1 and $1.5 \%$ over the entire portion of the trajectory for which the MEADS experiment was designed. The axial force coefficient uncertainty gets much greater at the low dynamic pressure ends of the trajectory. Again this uncertainty is likely overstated as the actual fit of the MEADS pressures to the LAURA CFD model are very good. The functional variation of $C_{A}$ uncertainty with dynamic pressure is more meaningful and should be considered when reading the results section. Future work will attempt to reconcile all the available data into the final reconstructed aerodynamic coefficients using a Kalman filter approach. The angle errors, both their variation along the timeline and absolute magnitudes are reasonable. As will be discussed below, it does appear that the flight MEADS reconstruction has measured these angles as accurately or better than these uncertaint bounds.

Reconciliation of Predicted and Reconstructed Aerodynamic Coefficients The MSL aerodynamic database uncertainties were modeled for use in Monte Carlo analysis to assess the sensitivities of the entry descent and landing system to off-nominal aerodynamic performance. The uncertainty model consists of a set of adders and multipliers applied to each aerodynamic coefficient [7]. The uncertainties were defined for three different flight regimes, rarefied/transitional, hypersonic continuum and supersonic continuum. For each Monte Carlo case, a random dispersion for each uncertainty value was applied, dispersing the aerodynamic coefficients by a constant fraction of the defined $3 \sigma$

\footnotetext{
${ }^{\dagger}$ Developed by the Centre National de la Recherche Scientifique (CNRS).

$\ddagger$ Available for download at: http://tftb.nongnu.org/
} 

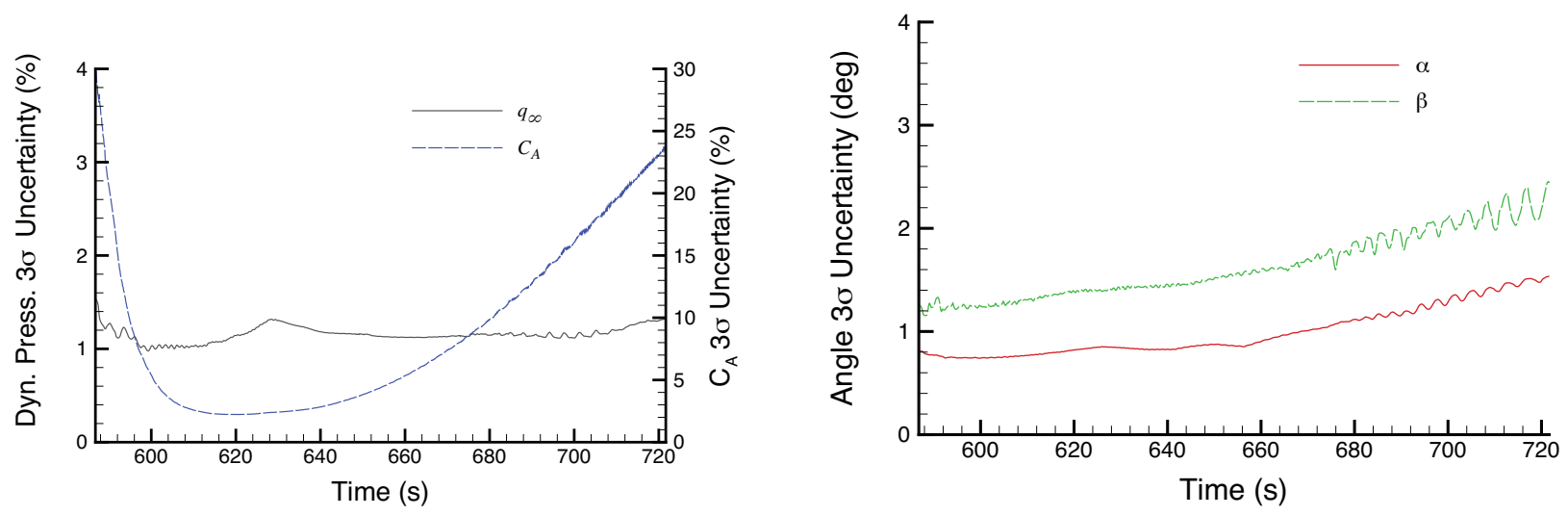

Figure 11. MEADS/DIMU uncertainties

Table 1. Static Aerodynamic Uncertainties

\begin{tabular}{cccccc}
\hline \hline Coefficient & $C_{A}$ & $C_{N}, C_{Y}$ & $C_{m}$ & $C_{n}$ & $C_{l}$ \\
& & & & & \\
Uncertainty & $U_{C_{A}}^{M}$ & $U_{C_{N}}^{A}, U_{C_{N}}^{M}$ & $U_{C_{m}}^{A}, U_{C_{m}}^{M}$ & $U_{C_{n}}^{A}, U_{C_{n}}^{M}$ & $U_{C_{l}}^{A}$ \\
\hline \hline Knudsen >0.1 & $\pm 5 \%$ & $\pm 0.01, \pm 10 \%$ & $\pm 0.005, \pm 20 \%$ & $\pm 0.005, \pm 20 \%$ & 0.0009 \\
Mach > 10 & $\pm 3 \%$ & $\pm 0.01, \pm 10 \%$ & $\pm 0.006, \pm 20 \%$ & $\pm 0.003, \pm 20 \%$ & 0.000326 \\
Mach <5 & $\pm 10 \%$ & $\pm 0.01, \pm 10 \%$ & $\pm 0.005, \pm 20 \%$ & $\pm 0.005, \pm 20 \%$ & 0.0004 \\
\hline
\end{tabular}

values for each flight regime. For example, consider the dispersion equation for the axial and normal force coefficient:

$$
\begin{gathered}
C_{A_{D i s p}}=C_{A}(\alpha, \beta)\left(1+U_{C_{A}}^{M}\right) \\
C_{N_{D i s p}}=\left[C_{N}(\alpha, \beta)+U_{C_{N}}^{A}\right]\left(1+U_{C_{N}}^{M}\right)
\end{gathered}
$$

Where $U_{C_{A}}^{M}$ is a multiplier applied to the nominal axial force coefficient, and $U_{C_{N}}^{A}$ and $U_{C_{N}}^{M}$ are adder and multiplier dispersions applied to the normal force coefficient. The $3 \sigma$ magnitudes of the adders and multipliers for each coefficient used in the MSL aerodynamic database are listed in Table 1 . The values were determined by comparisons of the database CFD solutions to wind tunnel data (heritage Viking wind tunnel data and new MSL test data) and recent flight data (Mars Pathfinder, Mars Exploration Rovers and Mars Phoenix). Sensitivity studies looking at gas models within the CFD codes, grid resolution, ablation and deformation, etc. were also conducted. Ultimately engineering judgment determined the final values as there are "unknown unknowns" associated with the flight vehicle flying through the Mars atmosphere that can not be accounted for prior to flight.

After comparing the aerodynamic database values, queried at the reconstructed trajectory states, to the MEADS/IMU extracted coefficients, a set of dispersion values that best reconciled the aerodatabase values with the reconstructed values were identified using least squares fits. This was done for the hypersonic and supersonic dispersions. The low density, transitional flight regime was outside the valid range of the MEADS transducers and that comparison is not assessed in this work. The identified dispersions give an estimate of how greatly the measured aerodynamic performance differed from the nominal preflight values.

\section{RESULTS}

The trajectory reconstruction results using DIMU and MEADS data are presented here and compared with the MSL aerodynamic database. First the angles of attack and sideslip are revisited with the reconstructed lift-to-drag 


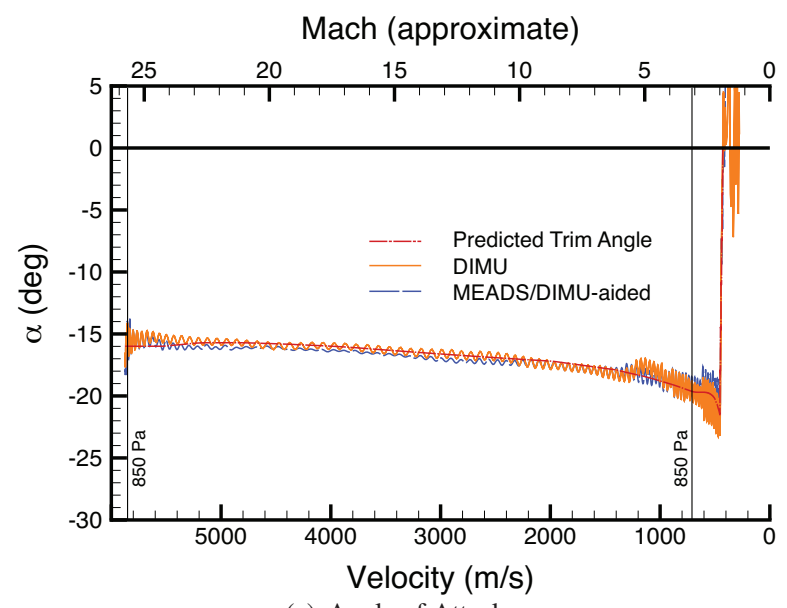

(a) Angle of Attack

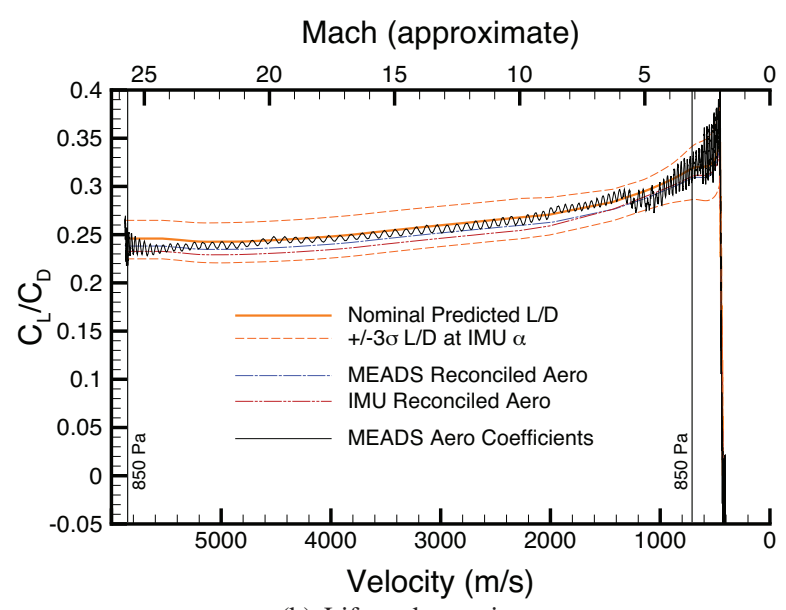

(b) Lift-to-drag ratio

Figure 12. Predicted and reconstructed trim angle-of-attack and lift-to-drag ratio

performance to show how the capsule flight performance compared to prediction. Then the individual aerodynamic coefficients are compared and assessed. Some qualitative comments supported by data from the reconstructions will be given to address some parameters that could not be extracted explicitly (dynamic stability, RCS aerodynamic interactions, etc.). This is the first assessment of the aerodynamic performance and much of the anomalies are subject to interpretation. The MSL project's best interpretations are provided, but more work is ongoing and may resolve some observed phenomena and anomalies in the future.

\section{Entry Performance}

Figure 12 shows the angle-of-attack and lift-to-drag performance during entry. In Figure 12a, the DIMU and MEADS/DIMU reconstructed angles of attack are compared to the preflight predicted trim angle (using the measured cg location and MEADS/DIMU Mach and velocity states along the trajectory). Note that in this figure and in many to follow the MEADS experiment boundaries $\left(q_{\infty}=850 \mathrm{~Pa}\right)$ are labeled. Both reconstructed $\alpha$ histories agree very will with preflight predictions. The DIMU data matches prediction almost exactly over most of the hypersonic regime. The MEADS/DIMU values differs by a constant value (less than a degree) over this range. There is a small departure in the DIMU $\alpha$ near Mach 6. This occurs at the end of the final bank reversal. The DIMU is unaware of the wind environment through which the capsule is flying; this angle departure is the consistence with the expected response of the DIMU if the capsule performed a bank reversal maneuver through a crosswind on the order 10 to 20 $\mathrm{m} / \mathrm{s}$.

Figure $12 \mathrm{~b}$ shows the lift-to-drag ratio determined from the MEADS dynamic pressure and accelerometer measurements, compared with the preflight prediction. The reconstructed performance was just slightly under predictions $(\sim 1-4 \%)$. This again is remarkable agreement over the entire entry and well within the dispersions used for trajectory design. As mentioned earlier, the dispersions were identified that best reconciled the aerodatabase forces with those reconstructed from flight. Lift-to-drag values using the parameters that best reconciled the coefficients using the MEADS/DIMU and DIMU angle-of-attack values are shown for comparison. It appears that reconciling the axial and normal force aerodynamics does not improve the agreement between the preflight and measured lift-to-drag ratios.

The sideslip reconstructions are plotted in Figure 13. In addition to the dynamic pressure boundaries, the SUFR maneuver is also noted in this plot. As the SUFR balance masses are jettisoned there are accelerations that introduce noise in the instrument measurements and small cg offsets that introduce sideslip dynamics. Reconciling the details during SUFR is beyond the scope of this work.

The radial cg offset is virtually zero and therefore no sideslip angle was expected. Both the DIMU and MEADS/DIMU reconstructions show a departure in sideslip angle reaching approximately $2^{\circ}$ at SUFR. There is currently no agreedupon explanation for this $\beta$ departure. As shown above, the MEADS uncertainties are larger than the beta values shown here. Some measurement error in port 6 or 7 could explain the MEADS divergence, although a DIMU di- 


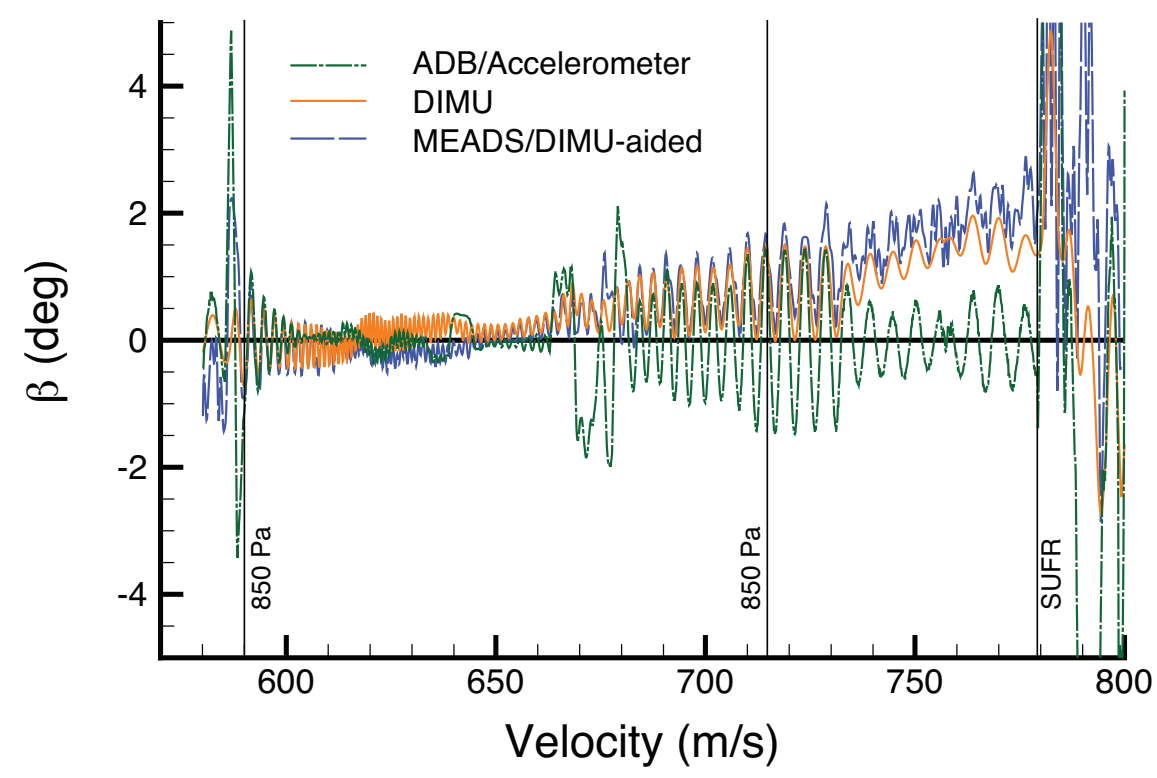

Figure 13. Comparison of angle-of-sideslip reconstruction results

vergence would not be expected to follow the MEADS results. A third attitude measurement method was added to provide more insight to this anomaly. The ratio of side force to axial force $\left(C_{Y} / C_{N}\right)$ was matched with the ratio of transverse and axial accelerations along the trajectory to find the sideslip angle using the MSL aerodatabase. This version of the reconstruction indicates that the accelerometers show no significant sideslip. It is hypothesized that the accelerations associated with the RCS firings during maneuvers introduced small orientation errors to the DIMU state which propagated, resulting in the DIMU sideslip divergence. It is curious that two semi-independent reconstructions both show similar beta departures, at odds with a third. In absolute terms, this departure is not a great concern for flight performance and the MEADS results are within the expected uncertainties. However, this anomaly remains an area of study.

\section{Static Forces and Moments}

Figures 14 through 17 show comparisons of the static forces extracted from the MSL flight trajectory with the MSL preflight aerodynamic database, queried at the reconstructed trajectory points. $3 \sigma$ aerodynamic database dispersions also plotted to show the uncertainty carried in preflight simulations. Figure 14 shows the axial force coefficient data. The aerodynamic database was queried at both the MEADS and DIMU angles-of-attack to show the variation due to error in determining the capsule attitude. That variation is small and the DIMU angle of attack history was selected for comparisons of MEADS and aerodynamic database coefficients and dispersions. In general there is very good agreement with the MEADS coefficient differing by just over $1 \%$ across much of the hypersonic regime. The disagreement is also close to constant across much of the entry. Figure 15a shows this data versus time across the hypersonic regime to show more detail.

The most notable $C_{A}$ disagreement occurs outside the MEADS dynamic pressure boundaries, leading up to the SUFR maneuver. The MEADS $C_{A}$ increases significantly in the low supersonic regime. Figure $15 \mathrm{~b}$ shows more detail of this MEADS $C_{A}$ anomaly. It was first thought that the backshell contribution to axial force was different than the Viking flight data and a modification to the modeled base correction might reconcile this anomaly. However, two supporting pieces of information suggest the axial force did not depart this much from prediction. First, the timeline of entry extended longer than predicted in this region (see Figure 6). It is suspected that the capsule flew through a reasonable tail wind $(10-20 \mathrm{~m} / \mathrm{s})$ or perhaps a region with atmospheric density lower than predicted. If the axial force were even greater than predictions, this would mean an even greater tailwind or density drop would be required to reconcile preflight trajectory predictions with the flight data. The second item that suggests the supersonic $C_{A}$ measurements are incorrect is the $C_{A}$ data itself just after the start of the SUFR maneuver. It appears that right at the first balance mass jettison, the $C_{A}$ data jumps back down in closer agreement with the aerodynamic database. The data 


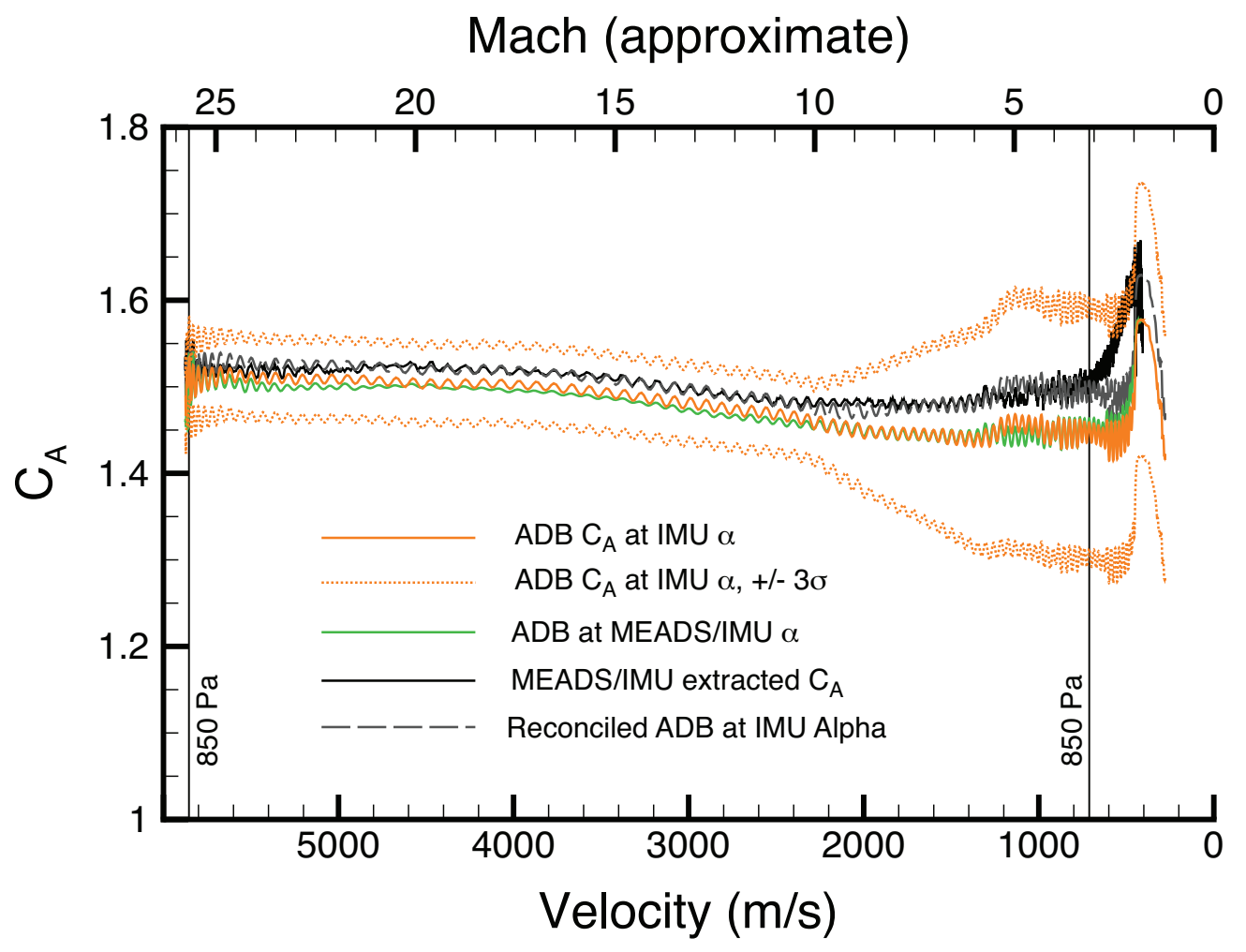

Figure 14. Comparison Aerodynamic Database $C_{A}$ to reconstructed values

is noisier there as the MEADS pressures are well below their intended ranges, but, it does appear that there is some kind of instrumentation or reconstruction issue with the data from about 700-780s. This error has not been resolved and remains an area of study. Again these discrepancies are within the MEADS uncertainties and overall preflight and MEADS data are in very good agreement.

Figure 16 shows the MEADS reconstructed normal and side force values compared to preflight predictions. The MEADS/DIMU data for both the normal and side force coefficients do noisier results, primarily because of noise in the accelerometer data and the normal and side force magnitudes are much smaller than the axial component. The RCS firings during bank reversals are also noticeable in the reconstructed coefficients. Overall there is excellent agreement between preflight prediction and these MEADS aerodynamic coefficients. The normal force coefficient is slightly smaller (more negative) than predicted. This to some extent mitigates the greater measured axial force coefficient resulting in the close lift-to-drag agreement shown above.

Figure 17 shows a comparison of the reconstructed oscillation frequency determined by TF analysis and preflight prediction using the local pitch and yaw slopes in the MSL database. The residuals are shown in Figure 17b with the slope uncertainties (multipliers) carried in the MSL database for reference. In general the agreement in frequency is good. Both preflight and reconstructed data shows the pitching frequency to be slightly higher than the yawing frequency. The variation in frequency, driven mainly by the dynamic pressure pulse, was predicted well by the MSL database, although the difference between the reconstructed values and prediction show a strong functional variation along the trajectory. This suggests an error in the TF analysis or the local static stability in the database. It was observed that the numerical differencing used in the TF analysis does tend to "shave off" the peak frequency near $625 \mathrm{~s}$. The peak frequencies are offset by a lag as well. This might suggest an issue with data filtering or some other numerical issue with the TF technique. It should also be noted that the $20 \%$ error carried in the MSL aerodynamic database was selected as a placeholder as there was very little data upon which to base the dispersions. Therefore, it is difficult to comment on how good this agreement is in terms of practical use in trajectory prediction and controller design. Future work should resolve the accuracy of the TF analysis. This analysis has shown agreement over most of the trajectory to within $10 \%$ or better and this may be reconciled to better agreement. This comparison is a good 


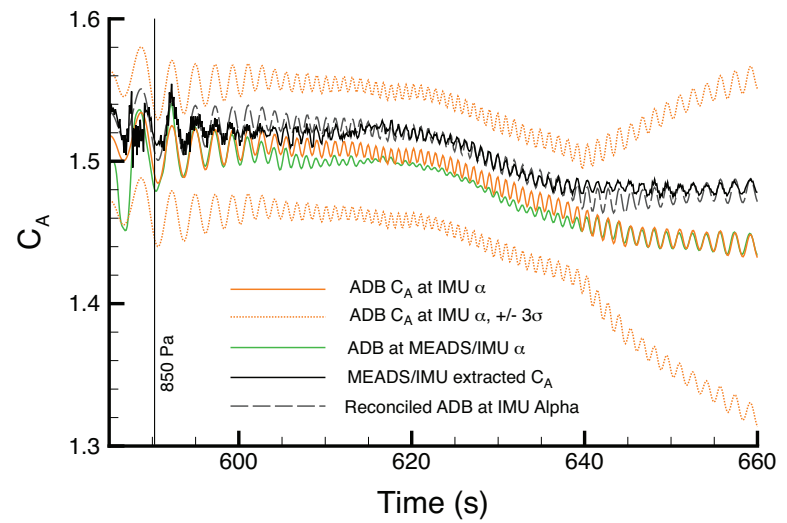

(a) Hypersonic detail

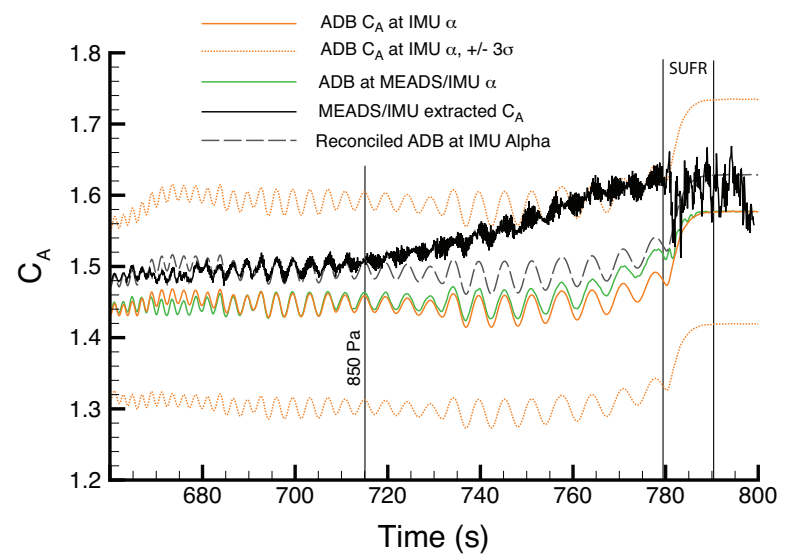

(b) Supersonic detail

Figure 15. Detail of $C_{A}$ comparison in hypersonic and supersonic regimes

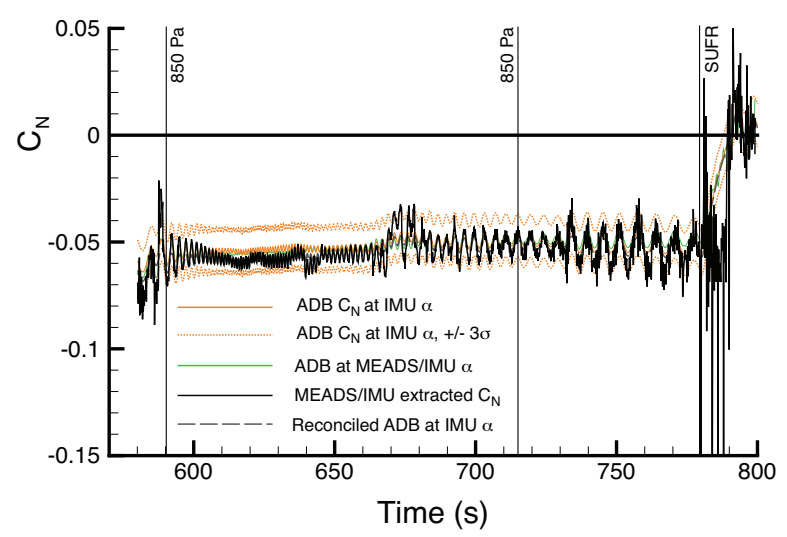

(a) Normal force coefficient

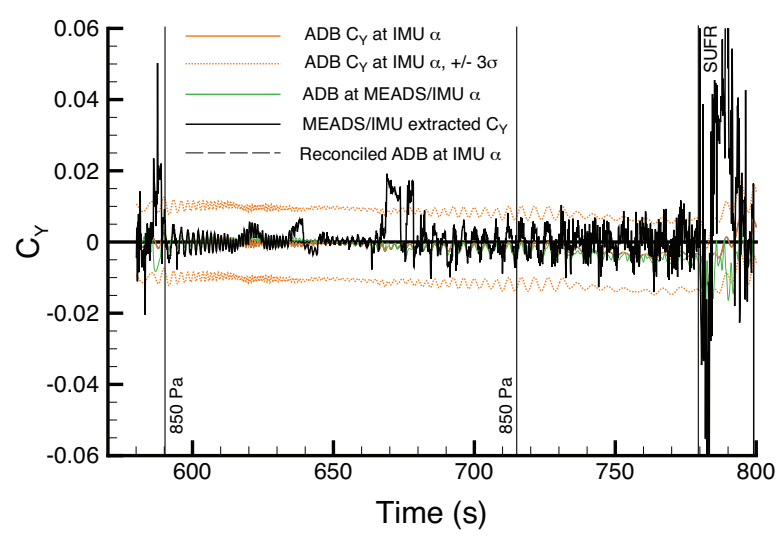

(b) Side force coefficient

Figure 16. Comparison of Aerodynamic Database $C_{N}$ and $C_{Y}$ to reconstructed values 


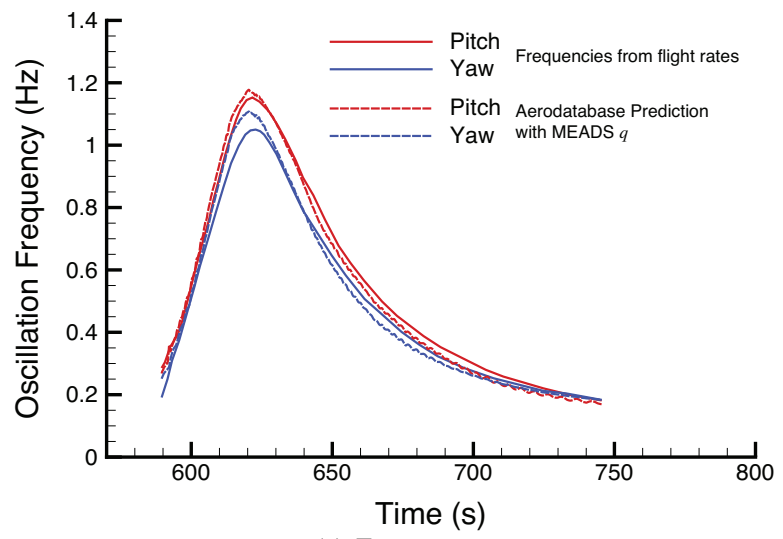

(a) Frequency

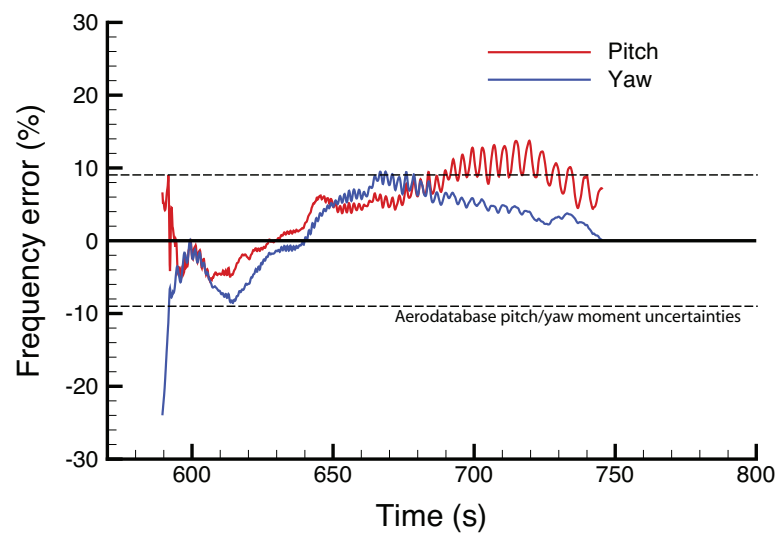

(b) Frequency residuals

Figure 17. Comparison of frequencies extracted from flight rates and those predicted by MSL aerodatabase

Table 2. Table of Dispersions Reconciling Aerodatabase and MEADS Aero Coeffs.

\begin{tabular}{|c|c|c|c|c|c|}
\hline Coefficient & Dispersion & Regime & $3 \sigma$ & Fit to DIMU & DIMU/3o \\
\hline \multirow[t]{2}{*}{$C_{A}$} & \multirow[t]{2}{*}{$U_{C_{A}}$} & Supersonic & $10 \%$ & $+3.28 \%$ & 0.3282 \\
\hline & & Hypersonic & $3 \%$ & $+1.08 \%$ & 0.3600 \\
\hline \multirow[t]{4}{*}{$C_{N}$} & \multirow{2}{*}{$U_{C_{N}}^{A}$} & Supersonic & 0.01 & -0.001274 & -0.1274 \\
\hline & & Hypersonic & 0.01 & -0.03285 & -0.3285 \\
\hline & \multirow{2}{*}{$U_{C_{N}}^{M}$} & Supersonic & $10 \%$ & 0.00698 & 0.0698 \\
\hline & & Hypersonic & $10 \%$ & 0.01634 & 0.1634 \\
\hline \multirow[t]{4}{*}{$C_{m}$} & \multirow[t]{2}{*}{$U_{C_{m}}^{A}$} & Supersonic & 0.005 & 0.00102 & 0.2056 \\
\hline & & Hypersonic & 0.006 & 0.00225 & 0.3748 \\
\hline & \multirow[t]{2}{*}{$U_{C_{m}}^{M}$} & Supersonic & $20 \%$ & $0.374 \%$ & 0.0187 \\
\hline & & Hypersonic & $20 \%$ & $0.056 \%$ & 0.0028 \\
\hline \multirow[t]{4}{*}{$C_{n}$} & \multirow[t]{2}{*}{$U_{C_{n}}^{A}$} & Supersonic & 0.005 & -0.00124 & -0.2497 \\
\hline & & Hypersonic & 0.003 & 0.00014 & 0.0471 \\
\hline & \multirow[t]{2}{*}{$U_{C_{n}}^{M}$} & Supersonic & $20 \%$ & $0.188 \%$ & -0.0094 \\
\hline & & Hypersonic & $20 \%$ & $0.006 \%$ & 0.0003 \\
\hline
\end{tabular}

starting point for future missions, when designing new guided entries.

Table 2 lists the aerodatabase dispersion identified by least-squares fit through the MEADS reconstructed coefficients. The "Fit to DIMU" columns represents the adder and multiplier dispersions that best reconcile the aerodynamic database with the MEADS coefficients using the DIMU angle-of-attack history. These fits used only the data within the MEADS 850Pa boundary so the large $C_{A}$ divergence does not skew the supersonic fit. Looking back at the comparison plots in this paper it is remarkable that each coefficient can be brought into good agreement across most of the trajectory with only two sets of dispersions for the two continuum flow flight regimes. It should be noted that the pitch and yaw moment multipliers are another bit of information for the assessment of the local static stability. These values show much closer agreement than did the TF analysis shown in Figure 17. Conversely the pitching and yawing moment adders are on the order of one standard deviation. These adder dispersons appear to be at odds with the prediction of trim angle which was in very close agreement with the DIMU attitude reconstruction. Future work will look more closely at the static stability information to establish a more complete story.

\section{Interpretation of RCS Firing History}

Some aerodynamic performance parameters could not be extracted explicitly. These include the dynamic stability characteristics, any adverse aerodynamic roll moments and aerodynamic interactions with the reaction control system 
jet plumes. For each of these items, a qualitative assessment can be made based on comparisons of the RCS firing history to preflight predictions. While not conclusive, this assessment is based on the interpretation of flight data after sifting through many many preflight predictions over the course if the MSL EDL development.

Aerodynamic Interactions with RCS A large development effort was undertaken during the MSL EV development to design and quantify the likely interactions of the RCS plumes with the surrounding wake environment $[18,19]$. The final interaction model used in simulation showed that RCS interactions would be small [20]. Also RCS interactions could only lead to excessive fuel usage if aerodynamic interactions exceeded control authority (control reversal). The flight data shows very little RCS activity at all except for the large bank reversals and minimal thrusting to maintain the desired bank angle within prescribed deadbands. There does not appear to be any excessive firing at the time of the bank reversals that would indicate the flight RCS torques differing from the design values significantly. It appears that the RCS interactions were small or perhaps beneficial (augmented RCS torques).

Dynamic Stability Schoenenberger et al [21]describe the ballistic range testing conducted for the MSL EV and how the extracted pitch damping coefficient, $C_{m_{q}}$, was implemented into the flight aerodynamic database. Due to faclity constraints, all MSL ballistic range testing was conducted with non-lifting models. The nonlifting pitch damping data were applied in a conservative manner in the MSL database, shifting the peak instability observed at $\alpha=0^{\circ}$ to the trim angle of attack. The unshifted non-lifting data indicated that the pitch damping coefficient would be dynamically stable at the MSL trim angle, but there was insufficient data to predict what the damping the lifting vehicle would experience. The flight data hints that shifting the damping data was overly conservative. Looking at preflight simulations, small oscillations were observed to grow in the supersonic regime exceeding pitch-rate deadband limits specified in the controller algorithm. Exceeding this limit prompted the RCS system to fire pitch jets to damp the oscillations. Looking at the flight rates and RCS history, the EV never exceeded the rate deadbands during capsule flight. This suggests the natural dynamic damping was greater than modeled in the aerodynamic database. The capsule appeared to act more like the non-lifting ballistic range data. As the angle oscillations are very small and the damping moments are only small modifiers to the static moments, it is very difficult to extract any pitch damping data explicitly. However, all available data suggests the RCS controller was not called upon to damp rates induced by any inherent instability.

Roll Torques Schoenenberger et al [7] showed how recession on the heatshield might lead to a pinwheel aerodynamic effect causing adverse roll moments. In off-nominal simulations, it was shown that roll torques could confuse the controller during bank reversals, causing the vehicle to fall off the guided trajectory and miss the desired landing ellipse. The RCS firing history complicates the search for adverse roll moments. A quiescent segment of the trajectory is necessary to identify any variation aerodynamic rolling moment. The frequency of RCS firing interrupts the "quiet" cruising of the MSL capsule. The longest segment of data near the peak dynamic pressure point indicates very little off-nominal roll torque. $1 \%$ of the $3 \sigma$ value listed in Table 1 is all that is required to replicate the minimal roll rate change over the quiescent segments. The minimal RCS firing activity away from the necessary bank reversals also suggests the controller was not fighting any anomalous aerodynamic moments.

\section{CONCLUSIONS}

The reconstructed aerodynamics compare remarkably well with preflight predictions. The trim angle of attack was predicted accurately and the reconstructed lift-to-drag ratio exceeded predictions by only a small percentage for most of entry. Reconciliation of the MSL aerodynamic database with MEADS/accelerometer aerodynamic coefficients showed that most parameters were in agreement with flight data to about $1 \sigma$ or better. It is also remarkable how well the data is reconciled across the entire entry using only the few uncertainty parameters in the MSL aerodatabase.

Two small anomalies have been identified and are being investigated further. First, the axial force coefficient extracted from the MEADS dynamic pressure and the DIMU axial accelerometer is much greater than preflight predictions in the supersonic regime leading up to balance mass jettison. This may be an instrumentation error and currently remains an area of study. The other notable anomaly is the divergence in sideslip across the entry. Both MEADS and DIMU angle reconstructions show a similar divergence, but this may be due to independent sources of error. The MSL aerodynamic database and the DIMU accelerometers were used to to reconstruct sideslip angle independent from the other methods. Those results indicate very small sideslip variation during entry. The MEADS angle uncertainties are greater than the sideslip angle reconstructed with the pressure data. The accelerations and vibrations introduced by RCS firings may cause orientation errors which propagate and accumulate over the entry. This is the other anomaly that continues to be investigated. 
Overall preflight aerodynamic predictions were excellent. The MSL aerodynamic database accurately predicted the flight performance and a database of similar fidelity could be used again with no improvements for a similar mission in the future. The RCS interactions and dynamic stability characteristics appeared to be benign with no obvious adverse effects on the flight performance. No unexpected roll torques were observed. Further analysis may be able refine the nominal aerodynamic data model for future missions, or may be able to justify reducing uncertainties to wring out additional performance from the MSL EV. Better pressure instrumentation especially in the low supersonic regime could resolve the axial force anomaly and perhaps the beta divergence. Better understanding of both of these phenomena, especially the supersonic axial force aonamly, could improve flight performance and decrease landing ellipse size.

\section{REFERENCES}

[1] N. A. Holmberg and R. P. Faust, "Viking '75 Spacecraft Design and Test Summary, Volume 1 - Lander Design," NASA Reference Publication 1027, 1980.

[2] R. N. Ingoldby, F. C. Michel, T. M. Flaherty, M. G. Doty, B. Preston, K. W. Villyard, and R. D. Steele, "Entry Data Analysis for Viking Landers 1 and 2," TN- 3770218, Martin Marietta Corporation, 1976.

[3] T. M. Flaherty, "Aerodynamics Data Book," TR- 3709014, Martin Marietta Corporation, 1972.

[4] S. Sell, "Powered Flight Design and Reconstructed Performance of the Mars Science Laboratory Mission," AAS 13-424, 2013.

[5] F. M. Cheatwood and P. A. Gnoffo, "User's Manual for the Langley Aerothermodynamic Upwind Relaxation Algorithm (LAURA)," NASA TM 4674, 1996.

[6] C. Riley and F. Cheatwood, "Distributed-Memory Computing with the Langley Aerothermodynamic Upwind Relaxation Algorithm (LAURA)," Advances in Engineering Software, Vol. 29, July 1998, pp. 317-324.

[7] M. Schoenenberger, A. Dyakonov, P. Buning, W. Scallion, and J. Van Norman, "Aerodynamic Challenges for the Mars Science Laboratory Entry, Descent and Landing," AIAA 2009-3914, 2009.

[8] A. A. Dyakonov, M. Schoenenberger, and J. W. Van Norman, "Hypersonic and Supersonic Static Aerodynamics of Mars Science Laboratory Entry Vehicle," AIAA 2012-2999, 2012.

[9] C. D. Karlgaard, P. V. Tartabini, R. C. Blanchard, M. Kirsch, and M. D. Toniolo, "Hyper-X Post-Flight Trajectory Reconstruction," Journal of Spacecraft and Rockets, Vol. 43, No. 1, 2006, pp. 105-115.

[10] C. D. Karlgaard, R. E. Beck, S. D. Derry, J. M. Brandon, B. R. Starr, P. V. Tartabini, and A. D. Olds, "Ares 1-X Trajectory Reconstruction: Methodology and Results," Journal of Spacecraft and Rockets, Vol. Accepted.

[11] C. D. Karlgaard, R. E. Beck, S. P. M. O'Keefe, S. A., B. A. White, W. C. Engelund, and M. M. Munk, "Mars Entry Asmospheric Data System Modeling and Algorithm Development," AIAA 2009-3916, 2009.

[12] M. M. Munk, A. Little, C. Kuhl, D. Bose, and J. Santos, "The Mars Science Laboratory (MSL) Entry, Descent and Landing Instrumentation (MEDLI) Hardware," AAS 13-310, 2013.

[13] C. D. Karlgaard, P. Kutty, J. Shidner, M. Schoenenberger, and M. M. Munk, "Mars Entry Atmospheric Data System Trajectory Reconstruction Algorithms and Flight Results," AIAA 2013-0028, 2013.

[14] C. D. Karlgaard, M. Schoenenberger, P. Kutty, and J. Shidner, "Mars Science Laboratory Entry, Descent and Landing Trajectory and Atmosphere Reconstruction," AAS 13-307, 2013.

[15] G. L. Brauer, D. E. Cornick, and R. Stevenson, "Capabilities and Applications of the Program to Optimize Simulated Trajectories (POST)," NASA CR 2770, February 1977.

[16] F. Auger and P. Flandrin, "Improving the Readability of Time-Frequency and Time-Scale Representations by the Reassignment Method," IEEE Transactions on Signal Processing, Vol. 43, No. 5, 1995, pp. 1068-1089.

[17] D. W. Way, J. L. Davis, and J. D. Shidner, "Assessment of the Mars Science Laboratory Entry, Descent, and Landing Simulation," AAS 13-420, 2013.

[18] D. A. A., C. E. Glass, K. T. Edquist, M. Schoenenberger, P. Chwalowski, J. Van Norman, W. I. Scallion, C. Tang, M. J. Wright, F. M. Cheatwood, B. R. Hollis, V. R. Lessard, and N. Takashima, "Design Considerations for Reaction Control Systems," 5th International Planetary Probe Workshop, Bordeaux, France, June 25-29 2007.

[19] A. A. Dyakonov, M. Schoenenberger, W. I. Scallion, J. Van Norman, L. Novak, and C. Tang, "Aerodynamic Interference Due to MSL Reaction Control System," AIAA 2009-3915, 2009.

[20] M. Schoenenberger, J. Van Norman, M. Rhode, and J. Paulson, "Characterization of Aerodynamic Interactions with the Mars Science Laboratory Reaction Control System Using Computation and Experiment," AIAA 20130971, 2013.

[21] M. Schoenenberger, L. Yates, and W. Hathaway, "Dynamic Stability Testing of the Mars Science Laboratory Entry Capsule," AIAA 2009-3917, 2009. 